\title{
On the scaling of three dimensional shock induced separated flow due to protuberances
}

\author{
S. Bhardwaj ${ }^{1}$, K. Hemanth Chandra Vamsi ${ }^{1}$ and R. Sriram ${ }^{1} \dagger$ \\ ${ }^{1}$ Department of Aerospace Engineering, Indian Institute of Technology Madras, Chennai, Tamil Nadu \\ 600036, India
}

(Received xx; revised xx; accepted xx)

Supersonic flow over 3-dimensional bodies protruding out of the turbulent boundary layer was investigated by means of experiments and numerical computations. A parametric study was performed by varying the shape and dimensions of the protuberance, as well as the freestream Mach number $(1.5,2,2.5,2.89,3.5)$. Surface streak line visualization, surface pressure measurements, and time-resolved schlieren visualization were employed along with RANS computations to elicit the complex flow features such as separation line, shock pattern, and the horseshoe vortex which greatly influences the flow dynamics in the separated region. The rise in surface pressure at mid-span due to separation (plateau pressure) was dependent only on the incoming flow parameters, and independent of protuberance geometry. The 2-dimensional free interaction theory, applied for normal shock induced separation, closely predicts the mid-span plateau pressure. Although protuberances are of varying shapes and dimensions, the inviscid bow shock (obtained from Euler computations) provided generalized scales, whose effects on shock boundary layer interactions are analyzed. The radius of curvature of the inviscid shock on the wall plane at the nose, which is theoretically related to the local second derivative (along the shock) of pressure jump, was found to be a determining parameter of mid-span separation length $\left(L_{\text {sep }}\right)$. Since the spanwise distance of the sonic point on the inviscid shock was found to be strongly correlated to its nose radius of curvature, it follows that the strong portion of the inviscid bow shock fixes the mid-span separation location. These observations concerning mid-span plateau pressure, and the role of strong shock portion in fixing mid-span separation, suggest that the $L_{\text {sep }}$ shall be predicted from a modification of the scaling laws for the length of plateau pressure region in 2-dimensional shock boundary layer interaction, with the inclusion of spanwise relieving effect. A correlation is obtained relating the $L_{s e p}$ with various incoming flow parameters and inviscid shock nose radius. The mid-span vortex core position was found to be linearly related to the $L_{\text {sep }}$. The radius of curvature of the separation shock is, however, found to be influenced by the entire inviscid shock, including the weak portion.

\section{Introduction}

Interaction of shock wave with boundary layer is an inexorable phenomenon in supersonic and hypersonic flights. Shock boundary layer interactions (SBLI) occur in almost all supersonic vehicles due to flow turnings and impinging shocks. Shock impingement and reflection at various locations in intakes, shock trains in isolators, flow over ramps, etc., are some common occurrences of SBLI. Of particular interest in this study are the interactions due to 3-dimensional objects (protuberances). SBLI due to 3-dimensional configurations such as swept ramps and protuberances are also frequently encountered in high speed flow fields. Protuberances on surfaces, flow field over which is the concern of the present study, may range from sub-boundary layer sized objects such as vortex generators used for flow control, to large scale wings and fins (on

$\dagger$ Email address for correspondence: r.sriram@iitm.ac.in 
fuselage) or turbine blades (on hub). Irrespective of the source of the shock and the configuration in which the interaction occurs, the phenomenon is basically the response of the boundary layer to the adverse pressure gradient imposed by the shock. A shock of sufficient strength can cause the separation of the boundary layer, which in turn results in generation of shock patterns in the outer inviscid flow (Délery 2011). These shocks can further interact with other surfaces. The interaction, especially if it results in flow separation, greatly alters the entire flow field and affects the performance of the system where they occur. Separation and reattachment also results in high local pressure and heat transfer. Such complicated flow features and the associated issues of practical interest have motivated decades of research on the interactions and their control (Délery \& Marvin 1986). Further, the SBLI is often observed to be inherently unsteady, exhibiting shock oscillations, with the incoming turbulent boundary layer also adding up to the unsteadiness. In the recent years, the understanding of unsteadiness and its mechanism has advanced greatly with the advancements in flow diagnostics and computations (Dolling 2001; Knight et al. 2003; Dussauge \& Piponniau 2008; Clemens \& Narayanaswamy 2014). However, the majority of the previous studies focus on 2-dimensional SBLI over canonical configurations such as impinging shocks, compression ramps, and forward-facing steps. Even in 2-dimensional configurations, 3dimensionality in the form of spanwise wrinkles in shock foot and other features is observed (Wu \& Martin 2008; Humble et al. 2009). With regard to the SBLI over 3-dimensional configurations, the knowledge of flow physics is limited, although such 3-dimensional interactions occur in many high-speed flow systems.

A schematic of the typical SBLI due to protuberances is shown in figure 1. The protuberance in the figure is cylindrical (with circular cross section), though the flow field with other shapes have qualitatively similar features as shown in the figure. In the present work, height and diameter/width of the protuberances are denoted by $h$ and $D$, respectively. The inviscid bow shock due to the protuberance has different shock strength at different spanwise locations on the plate, leading to a strong shock region in front of the body and a weaker shock away from it. This variation in the strength of the inviscid bow shock imposes spanwise varying adverse pressure gradient on the boundary layer. The 3-dimensional shock thus results in 3-dimensional separation of the boundary layer, and accordingly, the separation shock too is 3-dimensional. In the figures 1(a) and 1(b), the sonic points on the bow shock have been marked, which roughly gives the extent of the strong shock portion. $w_{s}$ is the distance of the sonic locations on the inviscid bow shock from the mid-span plane, while $h_{s}$ is the distance of sonic point on the reattachment bow shock from the base. $\Delta_{s}$ is the stand-off distance of the inviscid shock. The separation line forms just downstream of the separation shock foot, which marks the onset of the pressure rise on the base. The distance of the most upstream point on the separation line from the nose of the protuberance (measured in spanwise centre plane) is denoted as $L_{\text {sep }}$, referred simply as separation length. The reattachment of the separated shear layer on the protuberance results in 3-dimensional reattachment bow shock. The height of the 'triple' point at which the separation shock and the reattachment shock meets and interacts (along spanwise centre plane), is denoted as $h_{t p}$. The topology of the recirculating flow downstream of the separation line in the case of protuberance-induced SBLI is markedly different from those observed in 2-dimensional SBLI, and is expected to play a significant role in the flow dynamics. In 2-dimensional SBLI, for the time-averaged flow field, the separation bubble consists of closed streamlines, and the mass does not escape the bubble if not for shear layer entrainment. However, in the case of protuberance-induced separation, the recirculation region consists of a horseshoe vortex (orange band in figure 1(b)) in which the streamlines are free to escape sideways (Voitenko et al. 1966; Korkegi 1971; Sedney 1973). Another interesting observation concerning 3-dimensional SBLI is that they have higher peak pressures and heat transfer rates (locally) when compared with 2-dimensional SBLI (Burbank et al. 1962; Surber 1965; Hahn \& Frendi 2013).

The 3-dimensionality of the protuberance introduces more parameters than those in 2- 


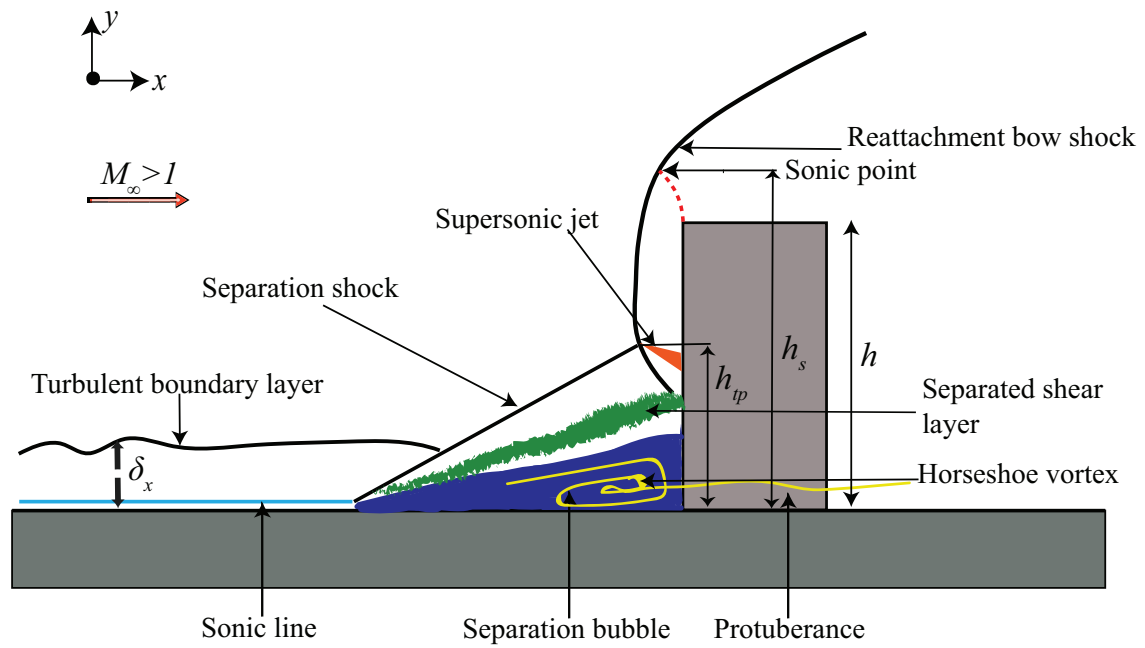

(a)

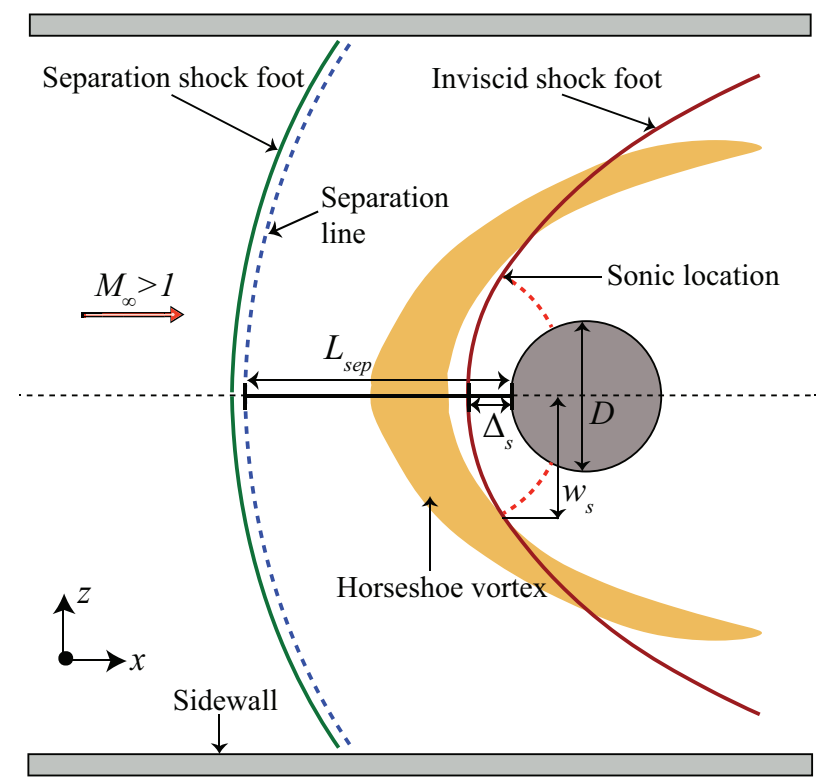

(b)

Figure 1: Schematics showing various flow features involved in shock boundary layer interactions due to protuberances in (a) spanwise mid-plane and (b) base plate wall surface.

dimensional SBLI. In 2-dimensional SBLI, the Mach number of the incoming flow, the incoming boundary layer thickness (typically measured immediately upstream of interaction) $\delta_{x}$, and the shock strength are the only parameters (not considering other influences such as wall heating or roughness). Although the parameters involved in 3-dimensional SBLI cannot be generalized due to the myriad 3-dimensional configurations, it is possible to study particular configurations such as protuberances. In the case of protuberances, particularly those considered in the present studies, which are symmetrical about the spanwise centered plane, other than Mach number and 
incoming boundary layer, the protuberance geometry introduces the key parameters: the shape, height, and spanwise width of the protuberance. The shape of the inviscid bow shock due to the protuberance depends on these parameters, which in turn affects the SBLI.

Early works concerning protuberance-induced SBLI largely focused on defining asymptotic height of the protuberance (mostly for cylindrical shaped protuberances), which is the minimum height beyond which increase in height had no effect on the separation bubble. In cylindrical protuberances which are of height comparable to or larger than boundary layer thickness, it was observed that $L_{\text {sep }}$ was only weakly dependent on the upstream boundary layer thickness (or Reynolds number), but was strongly correlated with the ratio of height to diameter, $h / D$ of the protuberance (Young et al. 1968; Sedney \& Kitchens Jr 1975, 1977; Dolling \& Bogdonoff 1981; Lindörfer et al. 2020) and from these correlations, it was possible to identify the $h / D$ beyond which the $L_{s e p}$ (or equivalently $h_{t p}$ ) did not vary. Voitenko et al. (1966) and Westkaemper (1968) presented some of the earliest empirical correlations relating $L_{\text {sep }}$ with $h / D$, at Mach numbers of 2.5 and 4.9 respectively; Westkaemper (1968) also presented a correlation for $h_{t p}$. Sedney \& Kitchens Jr (1977) reported extensive experimental data for various Mach numbers and cylinder dimensions; from the detailed parametric study, they presented correlations at various Mach numbers. They also reported some limited experimental data with protuberances of other shapes, noting that the flow filed with such shapes was qualitatively similar to that with circular cylinder protuberances. There are very few studies which concern non-circular protuberances. Voitenko et al. (1969) studied the effect of a change $\left(25^{\circ}\right.$ to $\left.170^{\circ}\right)$ in the angle of inclination of a rectangular-section protuberance (of 'infinite' height) on the shock structures and its impact on the flow patterns. The $90^{\circ}$ inclination case resembles the flat-faced protuberance case, one among a variety of protuberance shapes considered in the present study. The difference being, the height of the obstacle was effectively infinite, whereas, in the present study, a variety of heights are considered. Supersonic laminar flow over hemisphere was studied by Wang et al. (2012). The shock structures and 3-dimensional vortex structures downstream of the hemisphere were analyzed using Nanoparticle-based Planar Laser Scattering (NPLS) technique. From all these parametric studies, it can be concluded that most of the empirical correlations concern protuberances having the shape of circular cylinder and that for different Mach numbers, there are different correlations. Even among the class of 3-dimensional SBLI due to protuberances there are no universal parametric relations.

Pressure survey on the base plate along the centerline also reveals some markedly different trends when compared with surface pressure distribution for 2-dimensional SBLI. In 2dimensional SBLI, the pressure rises gradually as the flow separates, reaching a plateau in the separation bubble, after which it rises again to a peak value as the flow reattaches (Délery \& Marvin 1986). In the case of protuberance-induced SBLI too, the pressure rises as the flow separates, reaching a plateau. However, after the plateau region, the pressure drops to a local minimum before it rises to a peak pressure on the plate at the junction with the protuberance (Hung \& Buning 1985; Dolling \& Bogdonoff 1981). These alternating regions of high and low pressure are attributed to the horseshoe vortex (Hung \& Buning 1985; Ozcan \& Holt 1984). Along with the primary horseshoe vortex, presence of multiple vortices has been reported in the literature. Laminar supersonic flow over cylindrical protuberances was studied by Ozcan \& Holt (1984), where they observed three separation lines accompanied by three reattachment lines in front of the cylinder formed due to the presence of as many as six vortices in the flow. Sedney $\&$ Kitchens $\operatorname{Jr}$ (1975) also observed six vortices in front of the protuberance in some cases. The number of vortices formed was reportedly sensitive to the unit Reynolds number. Turbulent and high Reynolds number flows invariably have two vortex system: a horseshoe vortex or primary vortex in front of the protuberance and a secondary vortex at the junction between protuberance and base plate. The secondary vortex may not generally be captured in experimental visualizations due to its small size. In the numerical simulation of supersonic flow over the blunt fin, Hung \& 
Buning (1985) observed supersonic zones in the recirculation region in front of the protuberance (fin). One of them was present on the fin surface with Mach number reaching up to 1.2, while the other one on the flat plate with Mach number 1.4. The supersonic reverse flow on the protuberance surface is decelerated as it nears the plate junction, and this deceleration results in separation and secondary vortex. After the junction, the streamlines are again accelerated to supersonic speed. The observed surface pressure distribution too is thus due to these regions of intense acceleration and deceleration of the streamlines around the horseshoe vortex.

In 2-dimensional SBLI, the free interaction theory (Chapman et al. 1958) enables the estimation of pressure rise in the plateau region, incipient separation pressure, and plateau pressure. Further, the surface pressure measures feature in scaling laws for separation length, along with other flow parameters like Mach number and Reynolds number in 2-dimensional SBLI. By means of asymptotic analysis of the interaction region exhibiting triple-deck structure, the integration of the equations for lower deck (the balance between pressure and shear forces) resulted in scaling law for separation length (Burggraf 1975; Rizzetta et al. 1978; Davis \& Sturtevant 2000), which shall be discussed in detail later. The separation length was found to be related to the pressure term, typically expressed as the ratio of increase in surface pressure due to interaction to the pressure upstream of the interaction. However, unlike the scaling laws for 2-dimensional SBLI, the correlations concerning the SBLI due to protuberances are not universal in nature; as discussed before, for each Mach number there is a different empirical correlation, and the flow variables such as Mach number, Reynolds number or pressure ratio does not feature in these correlations. Threedimensional flows are highly complicated for such theoretical treatment. However, by using the extensive data generated for the present study (from experiments as well as computations), we seek to obtain some generalized scaling laws for 3-dimensional SBLI due to protuberances. The inviscid bow shock (obtained from Euler computations) provides generalized parameters accounting for varying shapes and sizes of the protuberance. Along with the shape and size of protuberances, Mach number is another parameter considered in this study. The effect of Reynolds number on the interaction is implicitly considered through the boundary layer thickness in this study, since the previous studies have suggested a weak dependence on Reynolds number for protuberances that are comparable to or larger than the boundary layer, as discussed before (Sedney \& Kitchens Jr 1977; Price Jr \& Stallings Jr 1967; Young et al. 1968; Korkegi 1971; Souverein et al. 2013). Experiments are performed with a nominal freestream Mach number of 2.87 in the supersonic wind tunnel at the Indian Institute of Technology, Madras. RANS computations are validated against the experimental data, and are used to obtain data as well as finer details of flow for a wider range of parameters. Using the experimental and computational data, we detail the flow topography, and obtain some generalized empirical correlations concerning the length scales of the protuberance-induced SBLI.

The overview of the paper is as follows. Section 2 describes the experimental and computational methodologies employed in the present work. Section 2.3 presents the validation of inviscid and viscous computations with the experiments performed at IIT Madras and empirical correlations available in the literature. In section 3, results obtained in the present study are discussed, and the flow features involved in the interaction are analyzed. The scaling of the protuberance induced SBLI is discussed and a correlation for the separation length (equation 4.8) is presented in section 4. Conclusions of the work are presented in section 5.

\section{Experimental and Computational Methodologies}

In the present study, experiments were performed in the facility present at the Department of Aerospace Engineering, IIT Madras. Computations with the same test section dimensions and freestream conditions were also carried out and were validated with experimental data. Computations with protuberances at few other Mach numbers were also validated using the 
reported data in the literature. Data for more protuberance shapes and freestream conditions were generated using computations for parametric studies. Computations also enabled an in-depth understanding of the flow features, which cannot be resolved using the limited experimental data. Details of the experimental and the computational methodologies, along with the validation of the computations, are presented in ensuing subsections.

\subsection{Experimental setup}

Protuberances of varying dimensions and shapes were exposed to a Mach 2.87 flow in the blow-down type wind tunnel present at Gas dynamics Laboratory at IIT Madras. The air supplied to the wind tunnel was dried by a refrigerated air dryer and subsequently compressed by a $100 \mathrm{HP}$ electric powered screw compressor. This compressed air was stored in storage tanks of $36 \mathrm{~m}^{3}$ capacity at 12 bar (gauge). In the settling chamber, stagnation pressure $\left(P_{\circ}\right)$ of 6 bar was maintained for all experiments; the stagnation temperature $\left(T_{\circ}\right)$ was room temperature of $\sim 300 \mathrm{~K}$. Air in the settling chamber was expanded through a contoured convergent-divergent nozzledesigned for a nominal Mach number of 2.87-into the test section, on which the protuberances can be mounted. The test section maintains a constant cross-section of $100 \mathrm{~mm} \times 113 \mathrm{~mm}$, with a streamwise length of $396 \mathrm{~mm}$. An image of the test section is shown in figure 2, with relevant dimensions and location of the protuberance placement marked. Static pressure $\left(P_{\infty}\right)$ in the test section was measured to be $19200 \mathrm{~Pa}$, by means of static pressure tap on the tunnel side wall. A traversable pitot probe placed at the streamwise location where the protuberances were to be mounted, was used for boundary layer survey. All the pressure measurements were carried out by using electronic pressure scanner, the details of which shall be presented in section 2.1.3. The wall-normal position of the pitot probe can be changed and fixed precisely by means of a screw gauge arrangement. Measurements using this pitot probe were taken in a step of $0.5 \mathrm{~mm}$, from wall-normal distances of $15 \mathrm{~mm}$ (in the freestream) to $2.5 \mathrm{~mm}$ (near the wall). The pitot tube was connected to the pressure scanner using tubes. The edge of the boundary layer was assumed at a point where flow velocity reached 0.99 times the freestream velocity $\left(U_{\infty}\right)$. Based on these measurements, we estimate the boundary layer thickness $\left(\delta_{x}\right)$ in the vicinity of the protuberance placement to be $7 \mathrm{~mm}$. The pitot measurement in the freestream, along with the surface static pressure on the tunnel wall was used to estimate the freestream Mach number. The freestream conditions and the boundary layer thickness measured at the location of placement of protuberance are presented in table 1 . The uncertainty in the measured quantity, calculated by performing uncertainty analysis described by Moffat (1988), has also been mentioned in the table.

Protuberances were placed mid-span on the bottom plate of the test section at a distance of $170 \mathrm{~mm}$ from the nozzle exit. Experiments were performed with rectangular-faced and cylindrical protuberances of two different heights: $15 \mathrm{~mm}$ and $30 \mathrm{~mm}$, as shown in figure 3 . The width of the rectangular-faced protuberance as well as the diameter of the cylindrical protuberance was $15 \mathrm{~mm}$. The face of rectangular-faced protuberance of height $15 \mathrm{~mm}$ is thus a square, and shall be referred as square-faced. More protuberance dimensions and geometries were employed in computations which shall be detailed in subsection 2.2.1.

For optical access, a $260 \mathrm{~mm} \times 120 \mathrm{~mm}$ glass window was present on the sidewalls of the test section. Surface pressure measurement and flow visualization techniques were employed to aid in the understanding of the flow physics. A detailed description of the flow visualization techniques is provided in the following sections. For reference, $x$ is in the direction of the flow, $y$ is in the bottom wall-normal direction, and $z$ is in the spanwise direction.

\subsubsection{Schlieren flow visualization}

The shock structures in the flow field and their unsteady dynamics are captured by means of time-resolved schlieren visualization technique. A conventional Z-type schlieren arrangement 


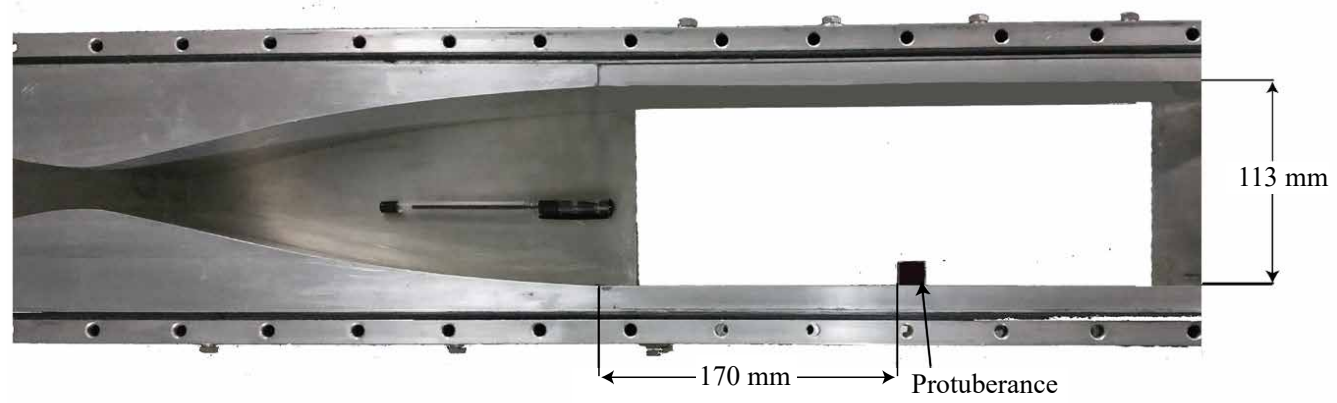

Figure 2: A photograph of the nozzle and test section, with a protuberance placed at $170 \mathrm{~mm}$ from nozzle exit, taken from the side with the sidewall removed.
$M_{\infty}$
$P_{\circ}$
$P_{\infty}$
(bar)
$(\mathrm{Pa})$
$T_{\circ}$
$U_{\infty}$
$(\mathrm{m} / \mathrm{s})$
$\underset{\left(\mathrm{kg} / \mathrm{m}^{3}\right)}{\rho_{\infty}} \quad \stackrel{\delta_{x}}{(\mathrm{~mm})}$
$2.87 \pm 4.4 \% \quad 6 \pm 1.1 \% \quad 19200 \pm 0.05 \% \quad 300 \pm 1 \% \quad 612 \pm 1.2 \% \quad 0.5979 \pm 14 \% \quad 7$

Table 1: Freestream flow conditions from the experiments.

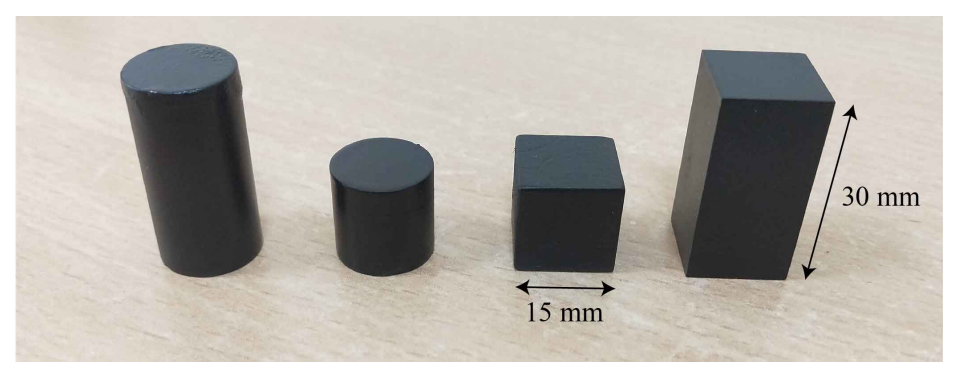

Figure 3: Different protuberances used in the experiments at Mach 2.87 flow.

was used in the present study. Halogen light source with two parabolic concave mirrors of focal length $2 \mathrm{~m}$ and $1 \mathrm{~m}$, a convex lens of focal length $53 \mathrm{~cm}$, and knife-edge were used for the schlieren flow visualization. A high-speed camera: Photron FASTCAM SA4 Model 500K-M1 was used for time-resolved digital imaging. Images were acquired at a rate of 30000 frames per second, with a spatial resolution of 448 x 240 pixels and shutter speed of 1/123000 s. Figure 4(a) and 4(b) shows instantaneous schlieren images of the flow field with cylindrical and square-faced protuberances respectively. The height of the cylinder is $30 \mathrm{~mm}$, while it is $15 \mathrm{~mm}$ for the squarefaced protuberance. The diameter/width of the protuberances is $15 \mathrm{~mm}$. The schlieren images show the side-view of the shock structures in the vicinity of the two protuberances. The flow features in figure 4(a) are essentially similar to those marked in the schematic shown in figure 1(a). The highly unsteady separation shock (S1) formed upstream of the recirculation zone interacts with the reattachment bow shock (B1), originating close to the protuberance. As observed from the schlieren images, the interaction between B1 and S1 is significantly different for the two cases shown in the figure. These interactions, classified by Edney (1968), lead to varying flow patterns and heat transfers to the surface of the protuberances. Shock oscillations and interactions shall be discussed in detail in later sections.

As a line of sight integrated information, the schlieren technique has limitations in resolving 


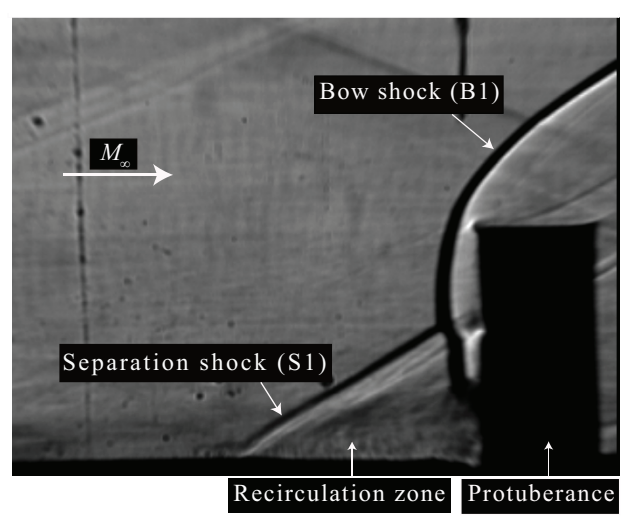

(a)

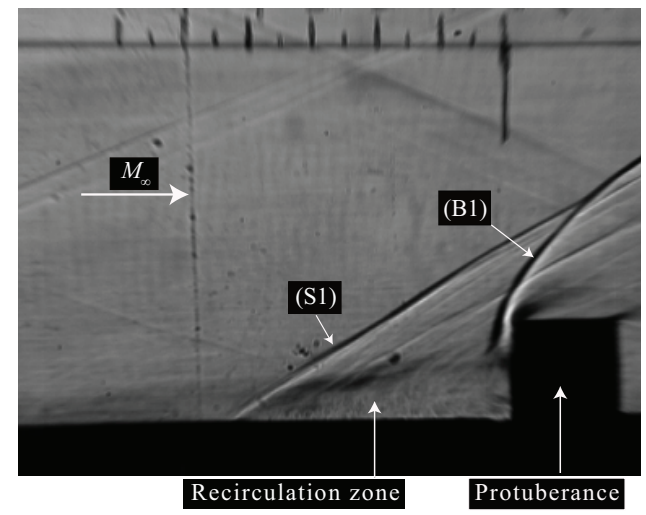

(b)

Figure 4: Schlieren flow visualization snapshots for (a) $30 \mathrm{~mm}$ height and $15 \mathrm{~mm}$ diameter cylinder and (b) square-faced protuberance of edge $15 \mathrm{~mm}$, with important flow features marked.

3-dimensional flows. Thus, the schlieren image of 3-dimensional flow has to be interpreted carefully. In the present case of SBLI due to protuberance, since the shock waves are typically bow-shaped with upstream most point (on any wall-parallel plane) located at the center of the span, the schlieren images effectively show the shock structure in the spanwise centered wallnormal plane. In order to resolve the spanwise picture of the flow field, oil flow visualization was employed to trace the surface streak lines on the base plate, the details of which shall be presented subsequently.

\subsubsection{Oil flow visualization}

Visualization of surface streak line pattern using oil flow technique is immensely useful in interpreting 3-dimensional flows and in particular separated flows. Although with the oil flow technique, only the streak lines on the base plate can be directly visualized, it provides insights on the overall 3-dimensional streamline patterns; and importantly, for the present study, it enables the tracing of the mean separation line, from which the separation length $\left(L_{\text {sep }}\right)$ can be measured. The mixture of oil was prepared using Titanium Dioxide $\left(\mathrm{TiO}_{2}\right)$ as the powdered pigment. Small proportions of Oleic acid were added as the dispersing agent, and SAE 30 oil was used as the base. The prepared mixture was spattered in the region of study using a brush, which resulted in numerous tiny drops of oil on the surface before performing the experiment. The size of the oil mixture droplets was negligible compared to the length scales involved in the flow, and their influence on the flow was minimal. The wind tunnel flow directs the oil to move in the direction of local wall shear forces, creating oil streaks. The streak line pattern evolves with the wind tunnel flow, and well before the end of the tunnel run time, it settles to a fixed pattern. The base plate of the test section was removed after every wind tunnel run, and the streak line pattern was recorded as a photograph. The $L_{\text {sep }}$ was also directly measured using scale. Further, a grid (of $5 \mathrm{~mm}$ spacing between two points) was drawn on the base plate before spattering with the oil. This helps in quantitatively tracing the mean separation line from the recorded photographs. Figure 5, shows the surface streak line pattern traced using oil flow technique for the flow over square-faced protuberance (side $15 \mathrm{~mm}$ ), illustrating how the mean separation line is traced and $L_{\text {sep }}$ is measured.

Excess oil mixture gets accumulated at the separation locations due to locally small shear stress values, during the tunnel run time. The oil flow patterns, especially at these separation locations, 


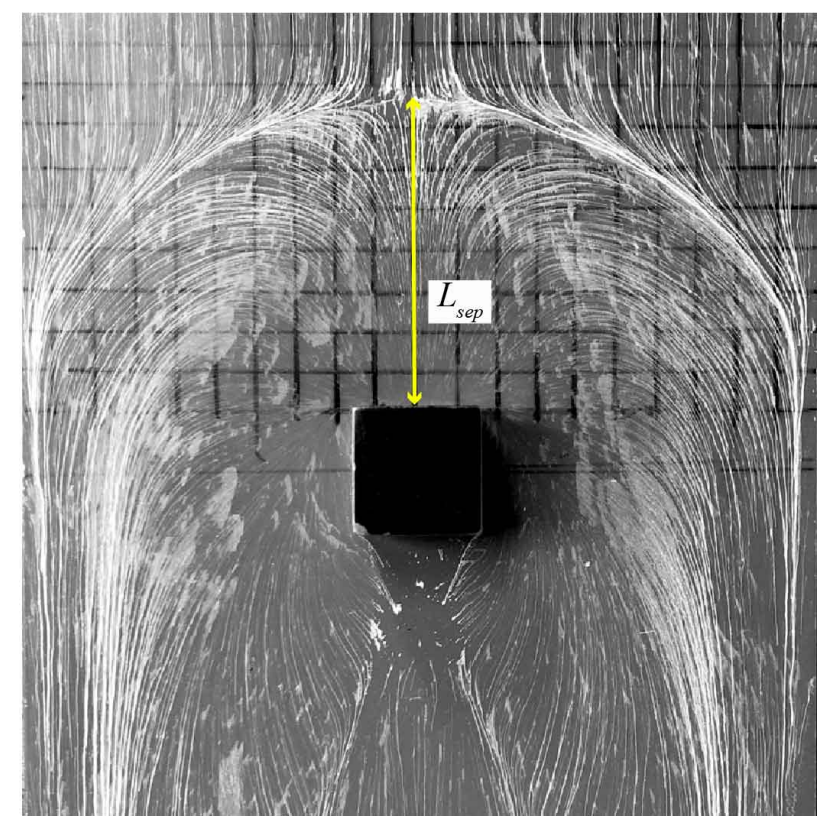

Figure 5: Surface streak line pattern on the base plate obtained through oil flow visualization for Mach 2.87 flow over square-faced protuberance of $15 \mathrm{~mm}$ side.

Measurement of the separation length is also illustrated.

have a tendency to smudge while closing the valve of the wind tunnel. This makes it difficult to accurately measure the length scales extracted in the oil flow patterns. To counter this, minimal quantities of the viscous oil mixture were used, which resulted in a sharp separation line (as seen in figure 5), with very little smudging due to the closure of the tunnel. However, for visualizing other features, experiments were performed with denser oil sprinkling; the streak line patterns extracted from some of those experiments shall be presented when discussing the details of the flow field.

\subsubsection{Surface pressure measurements}

Surface and pitot pressure measurements were made at different locations in the test section by means of an electronic pressure scanner: Scanivalve DSA 3217/16Px. The scanner gives a time-averaged value of the pressure for a maximum of 16 pressure ports at a time with a sampling rate of 500 samples/s. Freestream and boundary layer are also characterized by means of static pressure and pitot pressure measurements using the pressure scanner, as discussed before.

A detailed survey of surface pressure distribution caused due to protuberances was performed. For this, a base plate with 31 pressure taps of $0.6 \mathrm{~mm}$ diameter was used. Figure 6 shows the distribution of pressure taps on the base plate. This distribution was made particularly for the square-faced protuberance based on the regions identified from oil flow data, although the same distribution was used for measurements for SBLI with other protuberances. The details of the pressure distribution and the boundary layer profile shall be discussed along with the validation of computations in section 2.3.

\subsection{Computational setup}

Quantitative data obtained from experiments are limited to mean separation line and separation length, shock position, and time-averaged surface pressure values at discrete locations. Other information from visualizations are qualitative in nature. Moreover, an understanding of other 


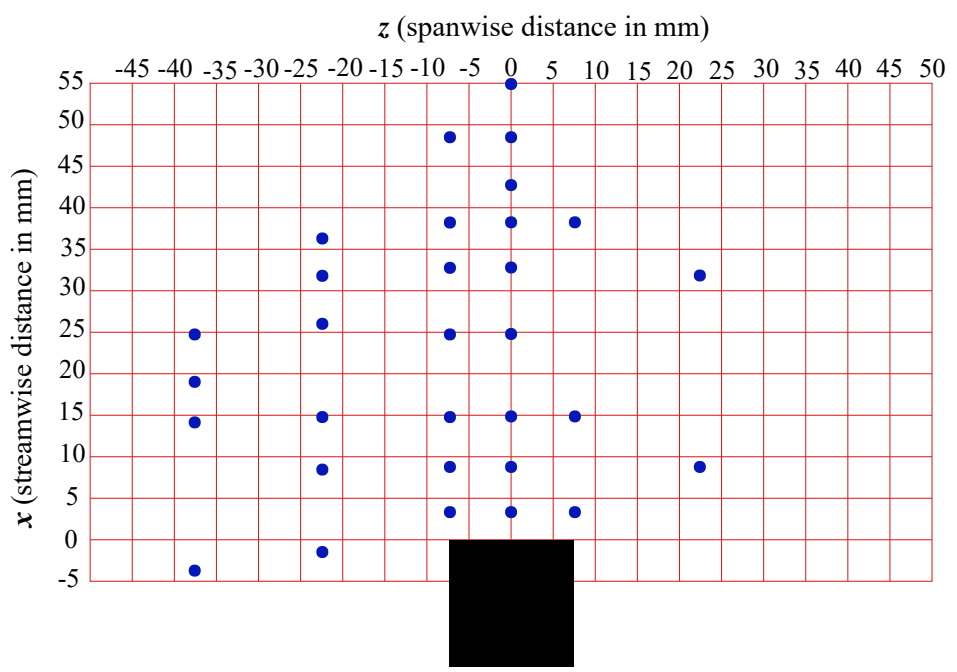

Figure 6: Distribution of pressure taps on the base plate for surface pressure measurements. The blue dots are the locations where the surface measurements were performed.

flow features requiring finer resolution of various flow quantities was difficult using experimental data. Therefore, along with experiments, extensive numerical simulations were performed with various geometrical and incoming flow parameters, which not only enabled resolution of finer flow features, but also provided the data for a detailed parametric study. Numerical computations were performed using CFD package ANSYS Fluent. The results of computations were validated with experiments by comparing the surface pressure data, separation line obtained from the oil flow visualization, and the mean shock structures obtained from schlieren. The computational results were also validated against the reported data in the literature. The details of the validation are given in section 2.3. Three-dimensional compressible Reynolds Averaged Navier Stokes (RANS) equations were solved numerically using the $k-\epsilon$ (Realizable) turbulence model. The computational domain consisted of a Mach 2.89 contoured nozzle exiting in a constant area duct of cross section $100 \mathrm{~mm} \times 113 \mathrm{~mm}$. The total length of the domain was $760 \mathrm{~mm}$. This domain (as shown in figure 7(a)) is similar to the test section in the experiments. Computations were also performed at other Mach numbers, which was achieved using different contoured nozzle geometries, exiting in the same test section cross-section of $100 \mathrm{~mm} \times 113 \mathrm{~mm}$. The grid over which computations were performed is shown in figure 7(b). All the protuberances were placed at the same distance of $170 \mathrm{~mm}$ from the nozzle exit. The grid was comprised of fine hexahedron cells close to 9 million in number. More details on the grid and the grid-independence study are presented in section 2.2.2. An implicit density-based steady solver was chosen for the computations with Advection Upstream Splitting Method (AUSM) flux formulation. Previous studies (Cappelli \& Mansour 2013; Lindörfer et al. 2017) have reported that the $k-\omega$ turbulence model over predicts the separation length in flows which involves strong SBLI, like in the present study. This over prediction of separation length was also observed in our earlier computations with $k-\omega$ model. Thus, the $k-\epsilon$ turbulence model was chosen, as it matched very well with the experiments performed. Courant-Friedrichs-Lewy (CFL) number was 0.3 in all the computational cases. Inlet and outlet of the computational domain were given pressure inlet and pressure outlet boundary conditions, respectively. At the inlet, stagnation pressure and temperature values were 6 bar and $300 \mathrm{~K}$, respectively, similar to experimental flow conditions. In order to avoid reflection 


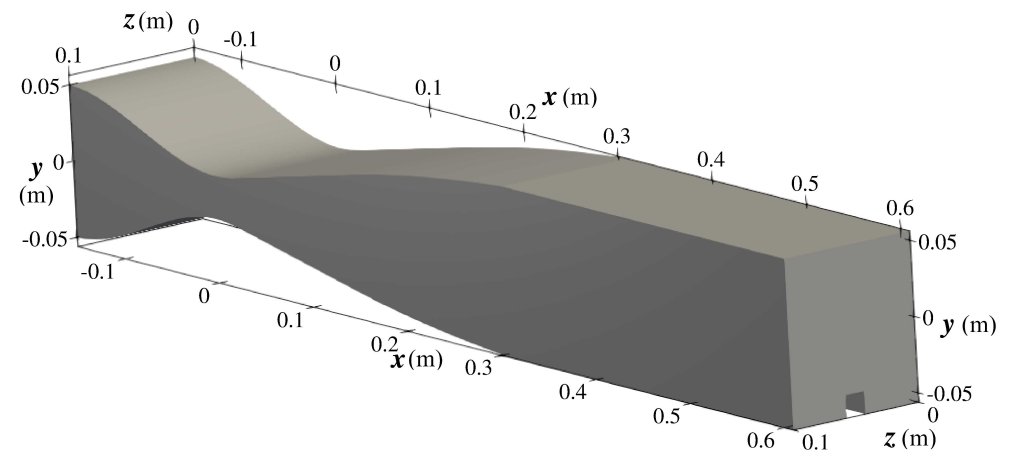

(a)

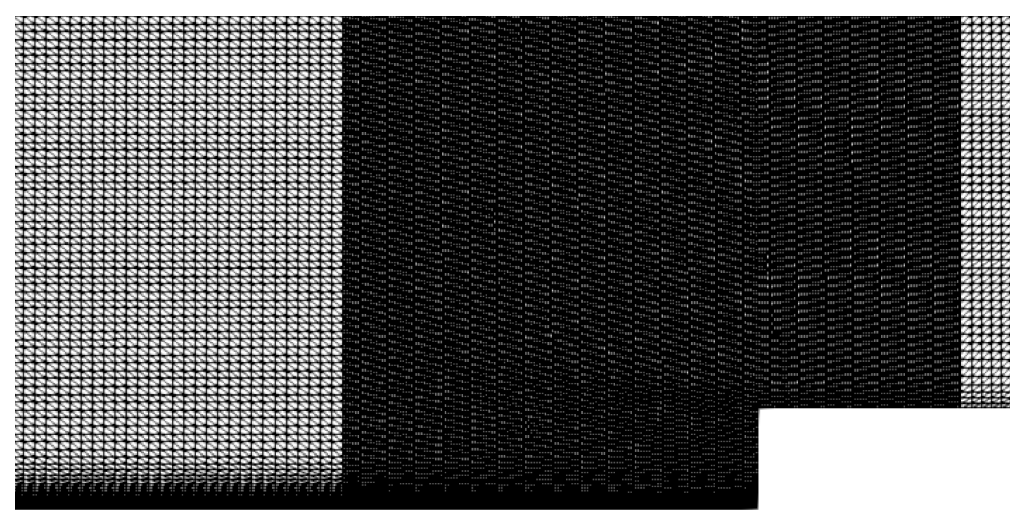

(b)

Figure 7: (a) Computational domain; (b) close up of the grid used for the steady computations of SBLI due to protuberances.

of shock waves back in to the domain, the outlet was given non-reflecting boundary condition. Other surfaces, viz. sidewalls, top wall, base plate, and the protuberance surface, were given adiabatic wall (no slip) boundary conditions. These boundary conditions of the computational domain resulted in a Mach 2.89 flow in the test-section. To avoid convergence issues due to reverse flow at outlet, the protuberance surface was extended to the outlet. The convergence of solution was assumed to have been achieved after ensuring the residuals were of the order $10^{-3}$ or lower for the equations being solved. Unsteady RANS (URANS) simulations were also carried out to capture the separation shock oscillations. It was found that the initial oscillations of the shock damped out to reach a steady state solution. The results of steady RANS computations matched very well with the evolved steady flow in the unsteady computations, as well as with the timeaveraged flow quantities obtained from experiments. Thus, the steady RANS computations are extensively performed to understand the time-averaged picture of the flow field and obtain scaling laws. A note on the shock oscillations shall be presented in section 2.4. Being a computationally intensive 3-dimensional problem, computations were performed in High Performance Computing Environment (HPCE) present at IIT, Madras.

\subsubsection{Protuberance geometries and Mach numbers}

Computations were performed for different cases to evaluate the effects of Mach number, shape, and dimension of the protuberance on the flow features. In the present work, flow over flat-faced, 


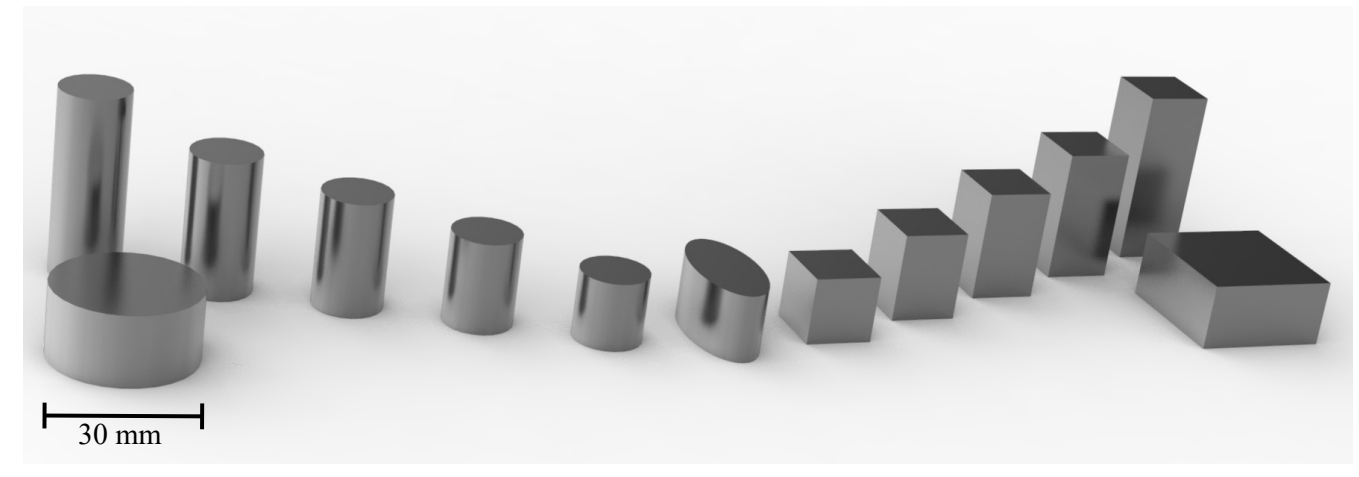

Figure 8: Different protuberance geometries used in the computations.

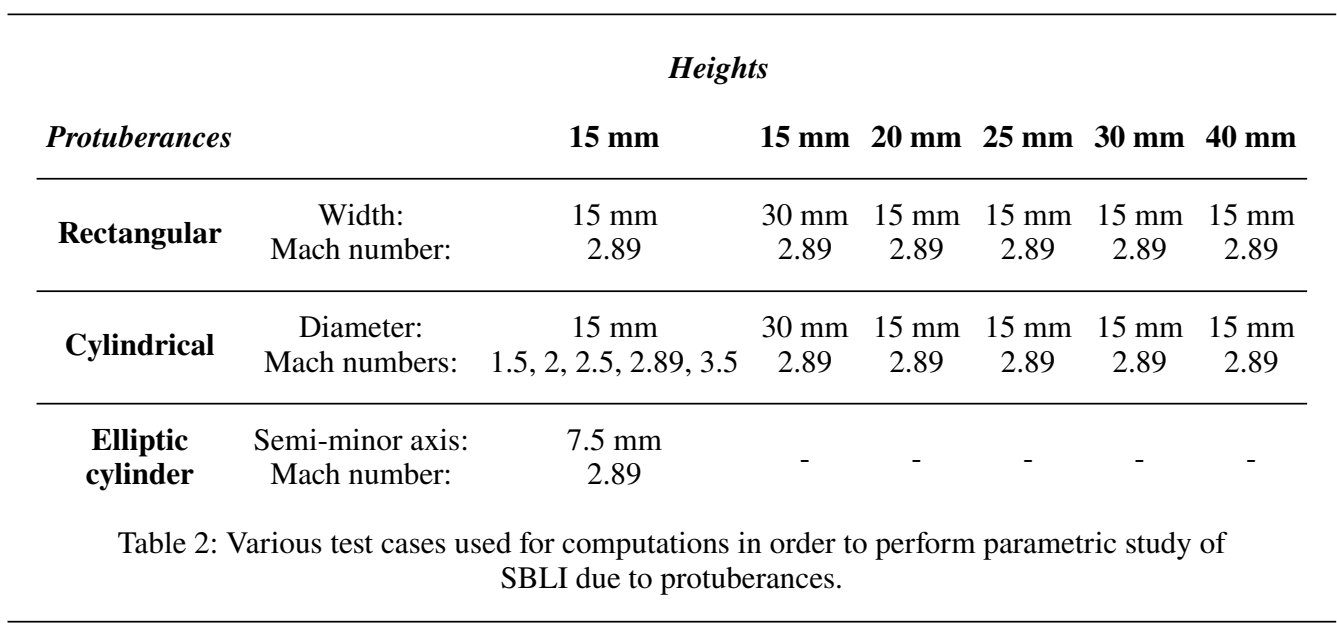

cylindrical, and elliptic protuberances was analyzed, and corresponding separation lengths were noted. The protuberances used in the numerical simulations are shown in figure 8. At Mach number of 2.89, in addition to the rectangular-faced and cylindrical protuberances used in the experiments (having width/diameter $15 \mathrm{~mm}$ ) of height $15 \mathrm{~mm}$ and $30 \mathrm{~mm}$, computations were also performed with protuberance heights of $20 \mathrm{~mm}, 25 \mathrm{~mm}$, and $40 \mathrm{~mm}$. Moreover, to include the effects of a change in the spanwise extent of the body, cylindrical and rectangular-faced protuberance of diameter/width $30 \mathrm{~mm}$ and height $15 \mathrm{~mm}$ were also employed. Additionally, an elliptic protuberance (height: $15 \mathrm{~mm}$, semi-minor axis: $7.5 \mathrm{~mm}$, and semi-major axis: $15 \mathrm{~mm}$ ) was also taken into consideration in the present work. Flat-faced protuberances have an infinite radius of curvature at the nose or stagnation point. In contrast, cylindrical and elliptic protuberances have a finite radius of curvature at the stagnation point. These differences in the curvatures of the bodies substantially change flow field and separation length scales. To develop a generalized scaling law for 3-dimensional SBLI, data for different Mach numbers was also considered. Mach 1.5, 2, 2.5, 3.5 flow was simulated for $15 \mathrm{~mm}$ height and $15 \mathrm{~mm}$ diameter cylindrical protuberance. Table 2 summarizes all the test cases used in the simulation.

\subsubsection{Grid-independence study}

Grid independence study was performed to check the sensitivity of the results on the mesh and to ensure that the results used for further analysis are independent of the grid size. To demonstrate this, the case of Mach 2.89 flow over square-faced protuberance of $15 \mathrm{~mm}$ edge was taken and 
five different grids with hexahedron cells were generated. Total number of cells in the domain were varied from 0.73 million to 22 million. The details of different grids used in the study are given in the table 3. For Grid C, Grid D, and Grid E, a uniform mesh element size was maintained in the constant area part of the computational domain. A finer mesh than Grid C (which has 5 million cells, with an edge length of $0.75 \mathrm{~mm}$ ) was generated by refining the mesh in the region near the protuberance, starting from $62 \mathrm{~mm}$ upstream of the protuberance till $30 \mathrm{~mm}$ downstream of it (well beyond the extents of the interaction zone for all the protuberance cases), resulting in 9 million cells for Grid B. This grid (shown in figure 7(b)), having an edge length of $0.35 \mathrm{~mm}$ in the refined portion near the protuberance, was used for all the computations in the present study. Lastly, to compare the results with extremely refined mesh, the grid was further refined in the vicinity of the protuberance, resulting in 22 million cells for Grid A with element edge length of $0.25 \mathrm{~mm}$.

To compare the results obtained for different meshes, flow properties were extracted at the base of the test section. Variation of coefficient of surface pressure $\left(c_{p}\right)$ and skin friction $\left(c_{f}\right)$ was compared for different grids at mid-span plane and $z / h=1$ plane. The results obtained for various grids used in the grid-independence study are shown in figure 9. Here, $x^{\prime}$ is the streamwise distance of a point from the front face of the protuberance. As can be seen from the figure, the curves for all the meshes, except for Grid E, align very well with the finest mesh (Grid A) results. Moreover, Grid A, Grid B, and Grid C with 5 million cells or more show minimal deviations in the profiles at the two different planes and accurately capture the separation point $\left(c_{f}=0\right)$, which is located close to $x^{\prime} / h=2.5$ in the spanwise mid-plane. The profile of the separation line was also compared for different grids used in the independence study, as shown in figure 10. In order to compare for different grids, the profile of the separation line was manually extracted from the surface streamline pattern obtained by using surface LIC plugin in Paraview. It can be observed that the separation line for all the grids except Grid E closely matches with each other with small deviations towards the sides. These deviations occur due to a less distinct separation line because of lower gradients, leading to a small error in picking the point of separation. In the figures 9 and 10, the results of the unsteady RANS computations, performed on a grid containing 5 million cells, are also shown. As discussed before, after a large number of time steps, the solutions of the unsteady computations reached a steady state, which is seen to match very well with the results of the grid independent steady computations.

Based upon the observations that the solution does not change with increasingly finer grid, the solution was assumed to be independent of the grid for Grid C and other finer ones. Hence, in order to optimize the computational cost and fidelity while maintaining a good spatial resolution, all the simulations performed in the present study had close to 9 million cells with the streamwise edge length of $0.35 \mathrm{~mm}$ near the protuberance. For all the grids used in the present computations, the $y^{+}$value was below 1 in order to resolve the features of the turbulent boundary layer close to the wall. From the validation tests, which will be presented in the next section, it was observed that the results of the computations on this grid showed good agreement with various data obtained in present experiments and empirical correlations present in the literature.

\subsection{Validation}

Properties of the undisturbed flow (without protuberance) in the test section at the location of protuberance placement were measured in the experiments and also extracted from the computational results. For the same test-section as in the experiments, the freestream Mach number of 2.89 was obtained in the numerical simulations. Freestream conditions and boundary layer thickness obtained from experiments were previously given in table 1 . The corresponding conditions obtained from computations are presented in table 4. From the computations, it was also possible to obtain the boundary layer displacement thickness $\left(\delta^{*}\right)$, which is also given in the table. A difference of $1.18 \%, 0.7 \%$, and $0.5 \%$ was noted for $P_{\infty}, M_{\infty}$, and $U_{\infty}$ between 
Number of cells (in millions)

Grid A 22

Grid B 9

Grid C $\quad 5$

Grid D $\quad 1.3$

Grid E $\quad 0.7$

Table 3: Various grids used for the grid-independence study.

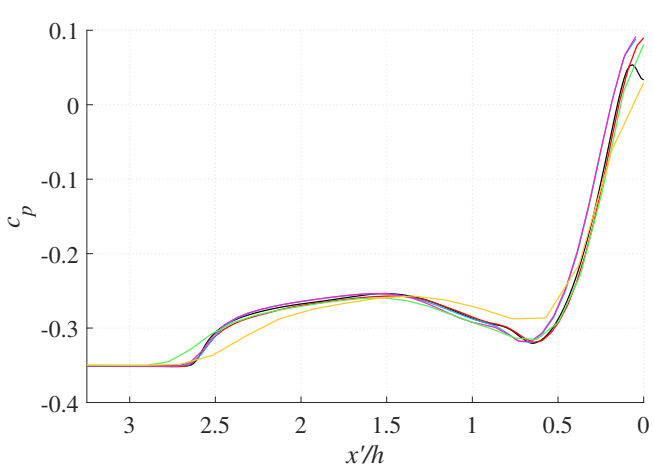

(a)

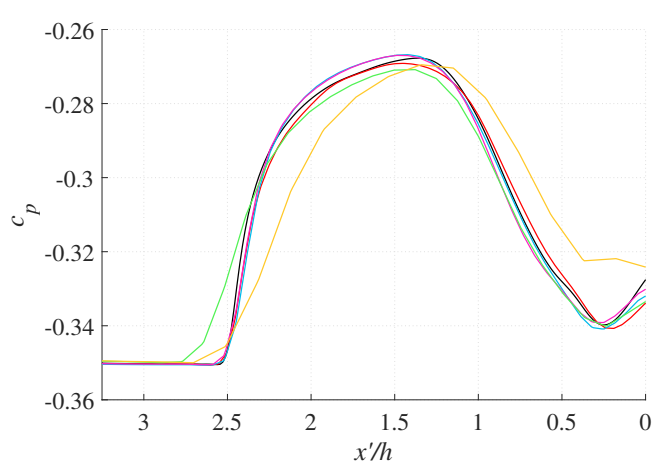

(c)

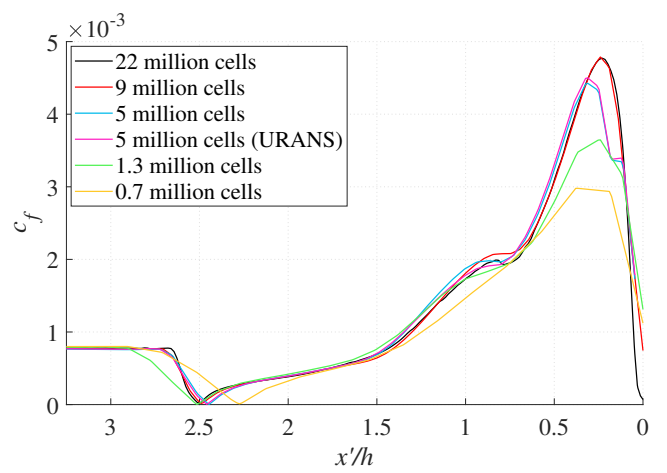

(b)

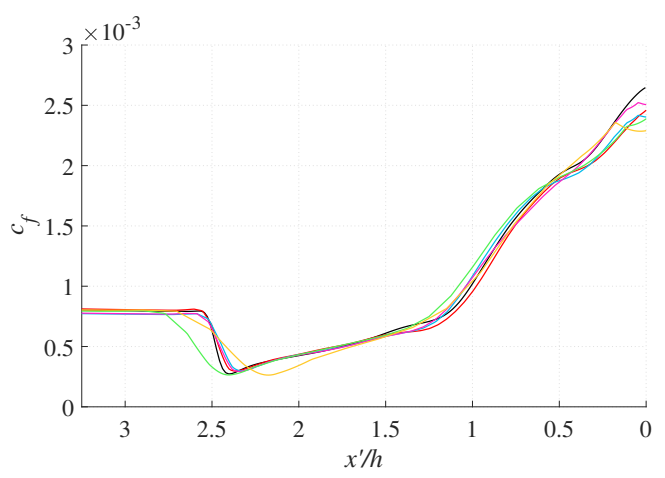

(d)

Figure 9: Comparison of the profiles of surface pressure $\left(c_{p}\right)$ and skin friction $\left(c_{f}\right)$ coefficients for different grids. (a) $c_{p}$ variation in mid-span plane, (b) $c_{f}$ variation in mid-span plane, (c) $c_{p}$ variation in $z / h=1$ plane, and (c) $c_{f}$ variation in $z / h=1$ plane.

experimental measurements and computations. Boundary layer being an important parameter in the present study on SBLI was characterized using the results. The profile of the turbulent boundary layer was also plotted using the velocity data in the computations and was compared with the pressure measurements performed using pitot rake in the experiments. Figure 11 shows the comparison for the same. As can be observed, a reasonable match of the two profiles was obtained. Boundary layer thickness at the location of protuberance placement was $7.18 \mathrm{~mm}$ for the undisturbed flow at Mach 2.89 in the computations, with a difference of $2.5 \%$ from the experiments. Boundary layer displacement thickness was calculated by extracting the velocity $(u)$ 


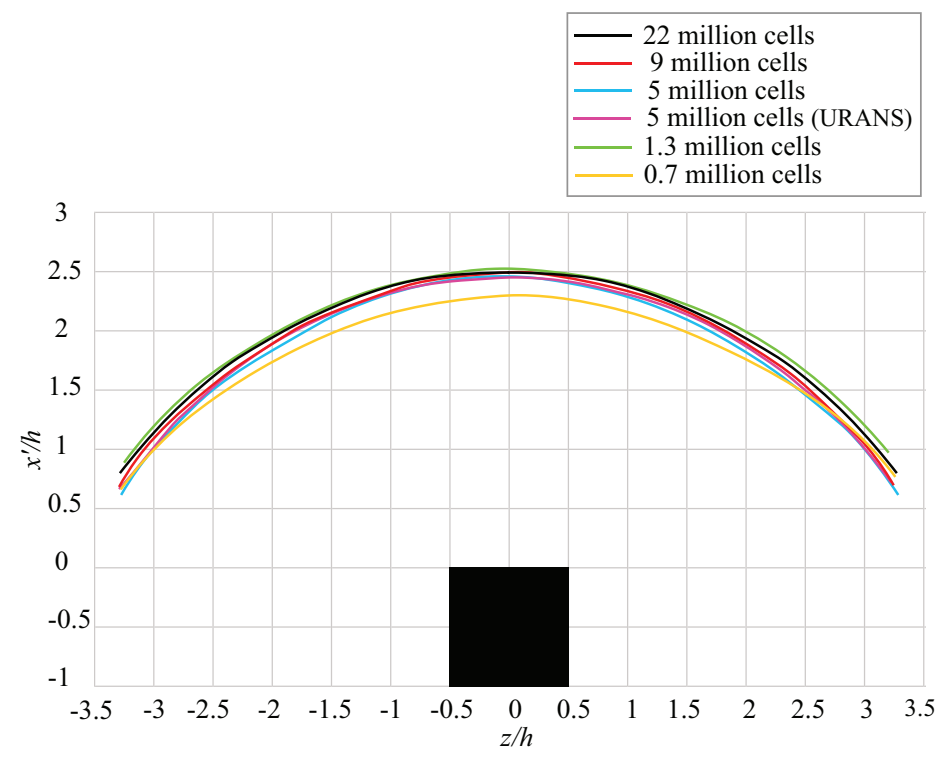

Figure 10: Comparison of the separation line profile for different grids.

$$
\begin{array}{ccccccccc}
M_{\infty} & \begin{array}{c}
P_{\circ} \\
(\mathrm{bar})
\end{array} & \begin{array}{c}
P_{\infty} \\
(\mathrm{Pa})
\end{array} & \begin{array}{c}
T_{\circ} \\
(\mathrm{K})
\end{array} & \begin{array}{c}
U_{\infty} \\
(\mathrm{m} / \mathrm{s})
\end{array} & \begin{array}{c}
\rho_{\infty} \\
\left(\mathrm{kg} / \mathrm{m}^{3}\right)
\end{array} & \begin{array}{c}
\delta_{x} \\
(\mathrm{~mm})
\end{array} & \begin{array}{c}
\delta^{*} \\
(\mathrm{~mm})
\end{array} & \begin{array}{c}
R e / L \\
\left(\mathrm{~m}^{-1}\right)
\end{array} \\
2.89 & 6 & 18972 & 300 & 615 & 0.5375 & 7.18 & 2.57 & 4.27 \times 10^{7}
\end{array}
$$

Table 4: Freestream flow conditions.

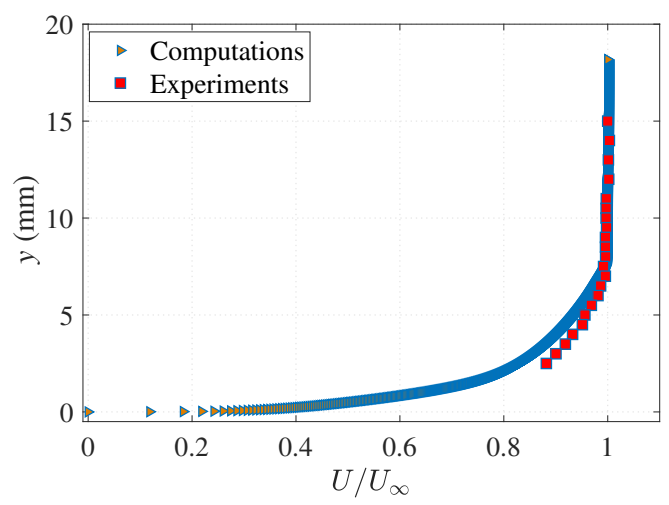

Figure 11: Comparison of the boundary layer profile obtained in the computations and the experiments.

and density $(\rho)$ data along a line perpendicular to the base plate, starting from the wall. A value of $2.57 \mathrm{~mm}$ for the $\delta^{*}$ was calculated based on the results.

Inviscid flow simulations were performed for each of the test cases mentioned in section 2.2.1 in order to obtain the inviscid bow shock in front of the protuberance, whose radius of curvature provides an important scale, as would be discussed in detail in section 4 . To validate the results 


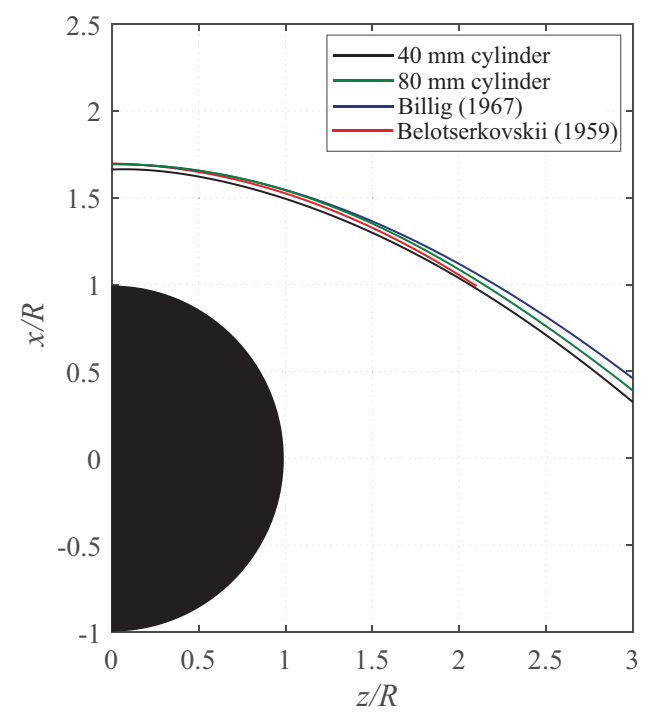

Figure 12: Comparison of the profile of the bow shock for the validation of inviscid flow simulations.

of the inviscid flow simulations, the profile of the bow shock as seen on the wall plane (which is a plane of symmetry for inviscid flow) was compared with the data and correlations reported in the literature. It has to be noted that many of the previous works have reported the bow shock profile for the 2-dimensional cylindrical cases. Since the study uses 3-dimensional protuberances, it is meaningful to compare the computational results of relatively longer protuberances with the 2dimensional correlations. Thus, inviscid computations over $15 \mathrm{~mm}$ diameter cylinders of $40 \mathrm{~mm}$ and $80 \mathrm{~mm}$ height were chosen for validation. Belotserkovskii (1959) presented computational data for inviscid flow over a cylinder at $M_{\infty}=3$, which was validated by the experiments with similar flow conditions. The profile of the bow shock at the wall plane was also compared with the relation presented by Billig (1967). The bow shock is approximated to be a hyperbola, whose profile - given as a relation between the coordinates ( $x$ and $z$ ) - is determined by the vertex radius of curvature $\left(R_{N}\right)$, shock stand off distance $\left(\Delta_{S}\right)$, Mach angle $(\mu)$ corresponding to the flow Mach number, and radius of the cylinder $(R)$. The relation is given as:

$$
x=R+\Delta_{s}-R_{N} \cot ^{2} \mu\left[\left(1+\frac{z^{2} \tan ^{2} \mu}{R_{N}^{2}}\right)^{0.5}-1\right]
$$

The shock profiles obtained from these different sources in the literature along with the profile of bow shock in the present computations for $M_{\infty} \sim 3$ are shown in figure 12. As can be seen, a good match is obtained with the profiles given in the literature. The shock profile almost overlaps with each other in the strong shock region of the bow shock. As expected, the $80 \mathrm{~mm}$ height cylinder shows better agreement with the 2-dimensional cases than the $40 \mathrm{~mm}$ height cylinder.

For the shock-induced separated flow field with protuberances, the separation line, surface pressure distribution and the shock pattern from numerical schlieren were compared with the experimental results. Firstly, the separation line obtained in the computations was compared with the one obtained in the surface oil flow pattern. Figure 13 shows the comparison for 15 $\mathrm{mm}$ diameter cylinder of height $15 \mathrm{~mm}$ and square-faced protuberance of edge $15 \mathrm{~mm}$. It can be observed that $L_{\text {sep }}$ in the computational results matches well with the experimental value. Moreover, the profile of the separation line closely follows the profile obtained in the experiments. 


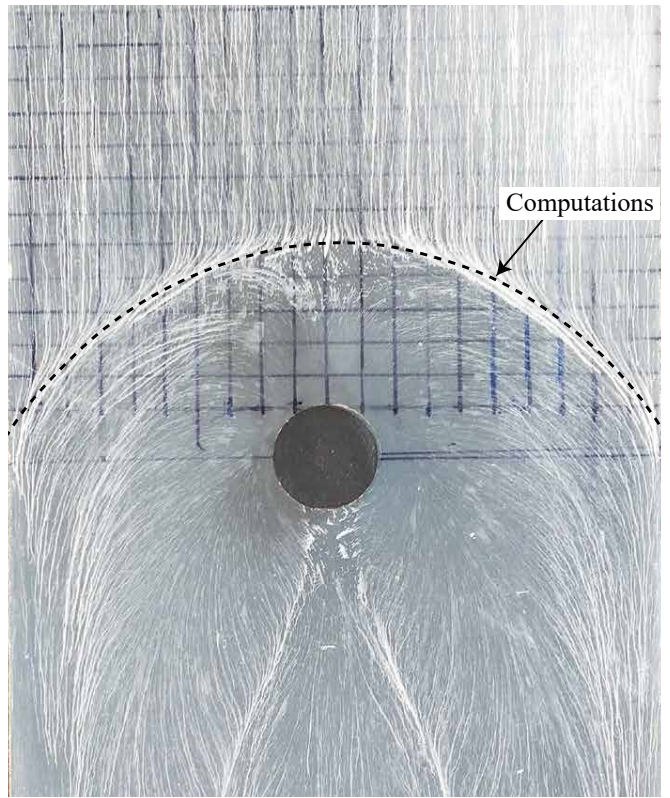

(a)

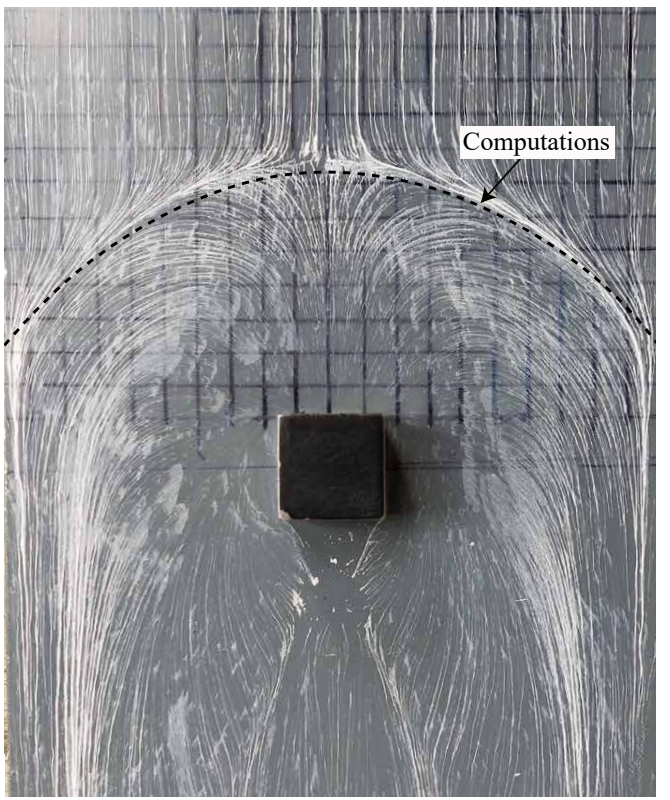

(b)

Figure 13: Comparison of separation line obtained from computations with oil flow visualization on the base plate for (a) $15 \mathrm{~mm}$ height and $15 \mathrm{~mm}$ diameter cylinder and (b) $15 \mathrm{~mm}$ square-faced protuberance.

The comparison was enabled by the use of an optimal amount of oil in experiments which resulted in a relatively sharp separation line.

Extensive pressure measurements were performed on the base plate to obtain the surface pressure distribution caused by the protuberance. The distribution of the surface pressure in the experiments, caused by the square-faced protuberance is shown in figure 14. The surface pressure is normalized by the freestream pressure. The diameter of the marker is made proportional to the magnitude of the pressure obtained in experiments at the location of the pressure tap. The dark dot inside the marker shows the accurate location of the pressure taps on the base plate. The dotted line marks the profile of the separation line obtained from the oil flow visualization. In the same figure, the difference (in percentage) between the surface pressure values obtained from the computations and experiments has also been mentioned. In most locations, the difference in the pressure values is within $10 \%$; the differences are high, particularly in places where there were sharp spatial variation in pressure. The highest differences can be observed at the location of the separation line. This can be attributed to the observation that the mean separation shock location in computations is slightly downstream for the square-faced protuberance than in the experiments. The unsteadiness of the separation shock, which is not captured in computations, may also have contributed to the differences at this location. Relatively higher differences in the values can also be observed at the locations where large pressure variations occur. Figure 15 shows the variation of the surface pressure in the interaction zone for square-faced protuberances; the pressure profiles on the wall along the lines $z=0 \mathrm{~mm}$ (mid-span) and $z=-7.5 \mathrm{~mm}$ are shown. For validation, corresponding data points obtained from experiments have also been marked, with error bar depicting $95 \%$ confidence interval of the property measured in multiple runs. Comparison of the variation of experimental and computed surface pressure along different lines at various spanwise locations i.e. $z=0 \mathrm{~mm}$ (mid-span), $z=-7.5 \mathrm{~mm}, z=-22.5 \mathrm{~mm}$ and $z=-37.5 \mathrm{~mm}$ for the 


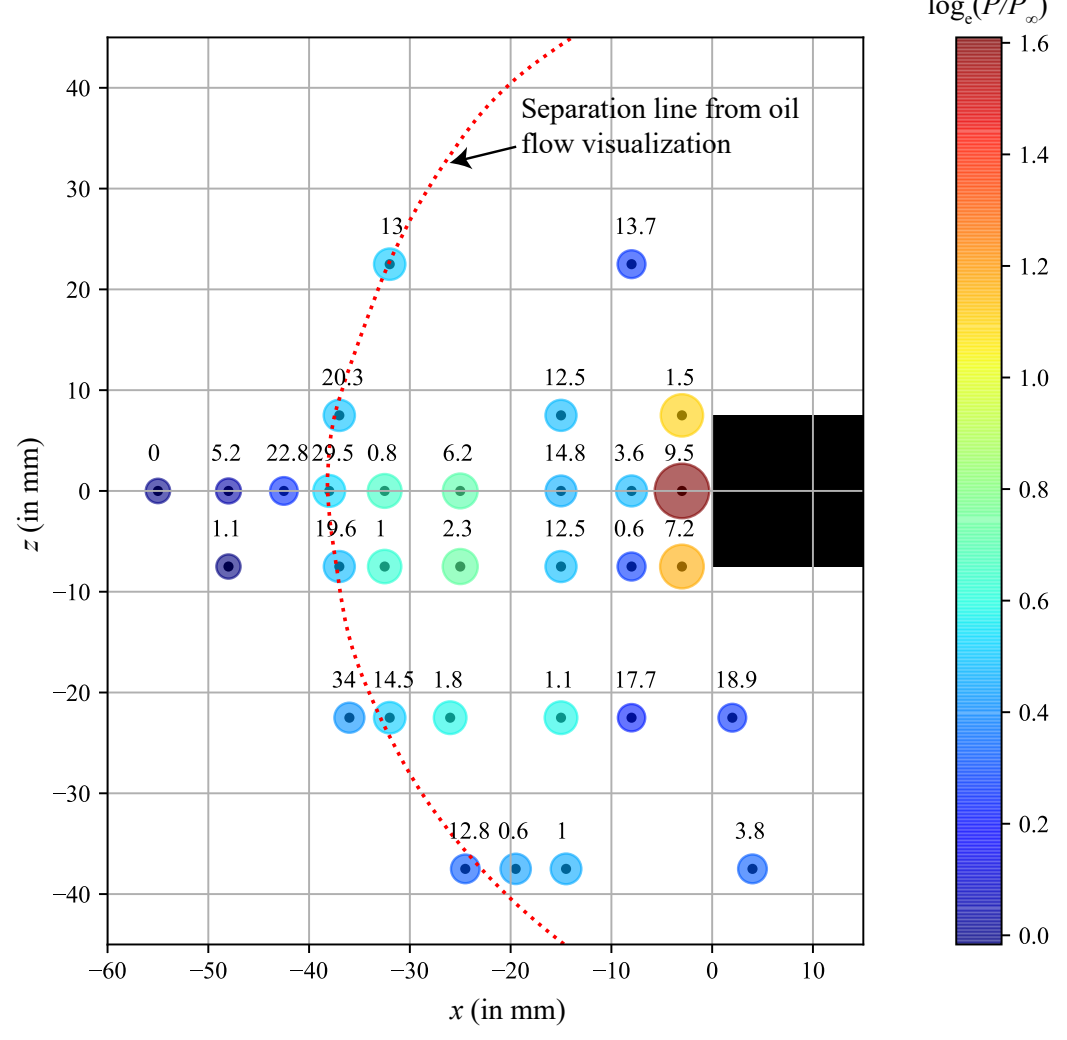

Figure 14: Surface pressure measured on the base plate in experiments along with the comparison with corresponding values in computations. The difference in the values between the computations and the experiments is shown above each marker. Separation line obtained from experiments has also been marked.

cylinder (15 mm height and $15 \mathrm{~mm}$ diameter) was also performed, as shown in figure 16 . The computational results can be seen to be in good agreement with the experiments.

Computations were also validated at other Mach numbers using an empirical correlation given by Sedney \& Kitchens Jr (1977). The authors reported a correlation for $L_{\text {sep }} / D$ variation with $h / D$ for cylindrical protuberances at Mach 2.5 and 3.5 incoming flow. Figure 17 compares the computational results of the present study with the correlation, which assumes an asymptotic functional form of the variation for large $h / D$. An excellent match can be observed with little deviation from the correlation.

The numerical schlieren obtained from steady computational data was compared with the mean shock structures from the time-resolved schlieren data. In order to obtain the mean location of the separation shock and also to get a statistical sense of the flow field, averaged schlieren image was developed by taking 3000 instantaneous schlieren images captured at $30000 \mathrm{fps}$. The pixel color in a grayscale image varies from black (pixel intensity value of 0 ) to white (pixel intensity value of 255), and the rest of the pixels taking intermediate values. The pixel intensity data of all the individual images was extracted and averaged in time. Consequently, the mean locations resulted in the brightest portions of the averaged schlieren image. A standard deviation $(\sigma)$ of the data was calculated, and the averaged schlieren image was colored accordingly. The resulting averaged schlieren image for $15 \mathrm{~mm}$ height square-faced protuberance is shown in figure 18. The dashed line highlights the separation shock in the averaged picture. For comparison, separation shock 


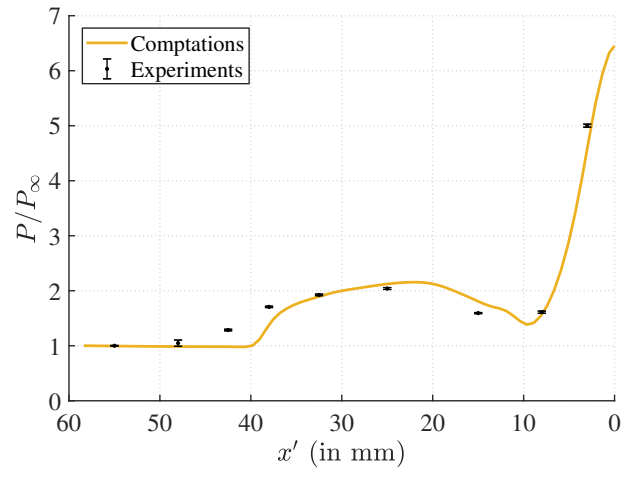

(a)

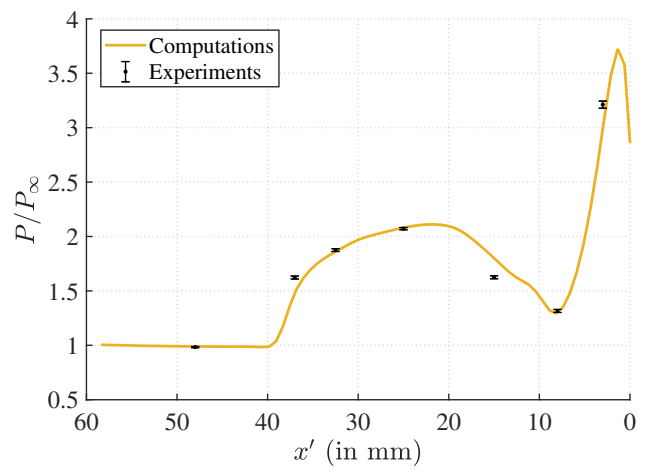

(b)

Figure 15: Comparison of surface pressure profile obtained in computations with the experiments for square-faced protuberance at (a) $z=0 \mathrm{~mm}$ plane (mid-span) and (b) $z=-7.5 \mathrm{~mm}$ plane.

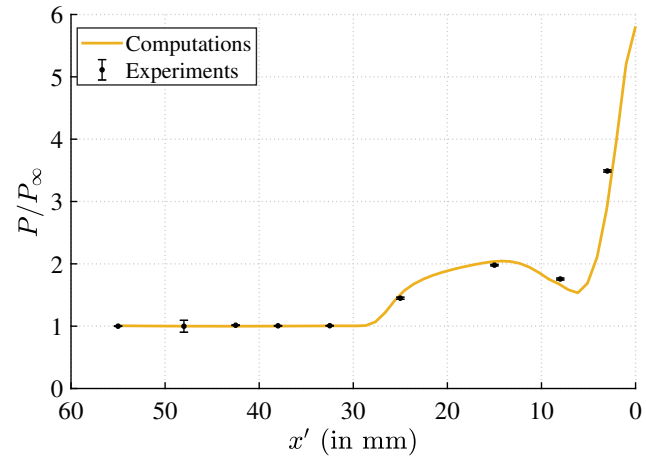

(a)

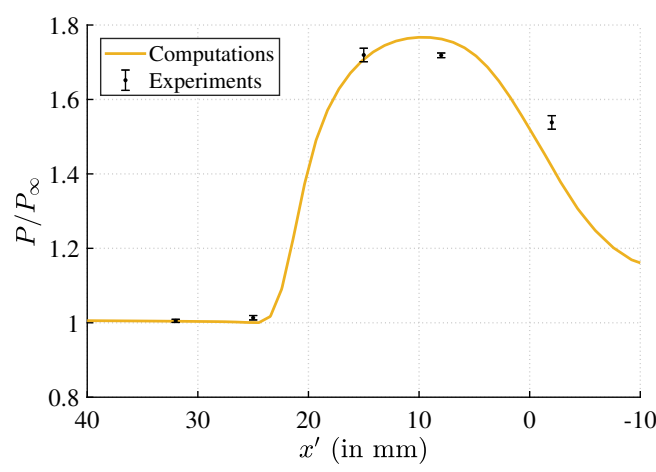

(c)

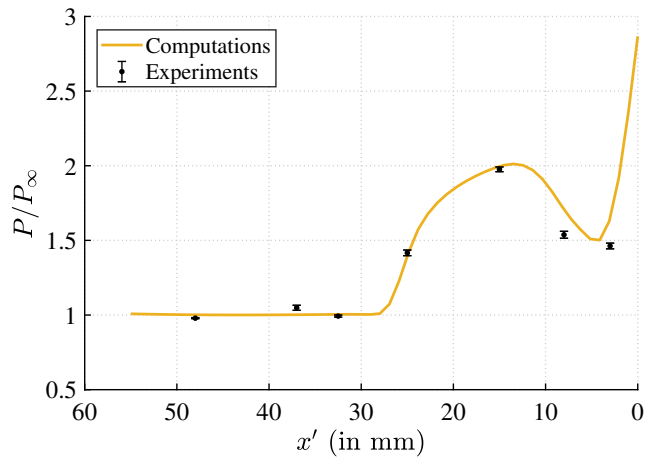

(b)

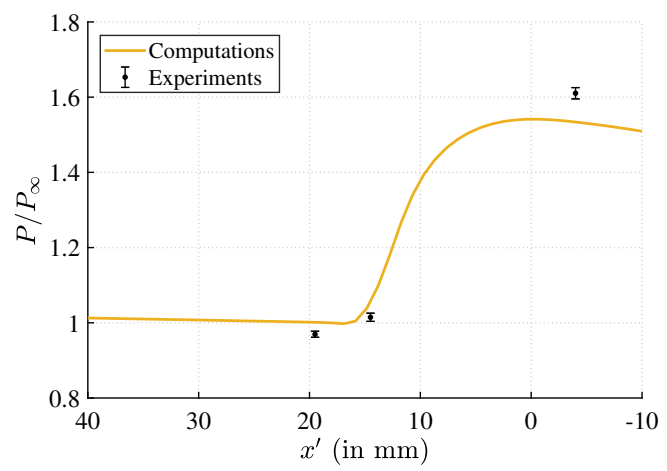

(d)

Figure 16: Validation of computational results with the experiments performed for $15 \mathrm{~mm}$ height and $15 \mathrm{~mm}$ diameter cylinder. Pressure was surveyed on the base plate of the test section along a line with spanwise locations: (a) $z=0 \mathrm{~mm}$ (mid-span), (b) $z=-7.5 \mathrm{~mm}$, 


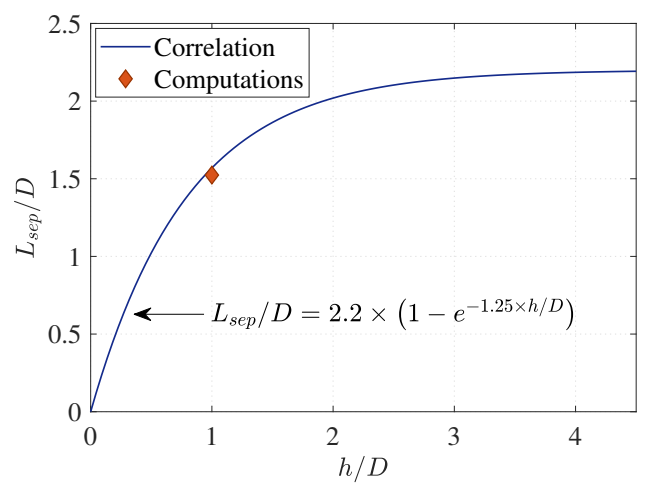

(a)

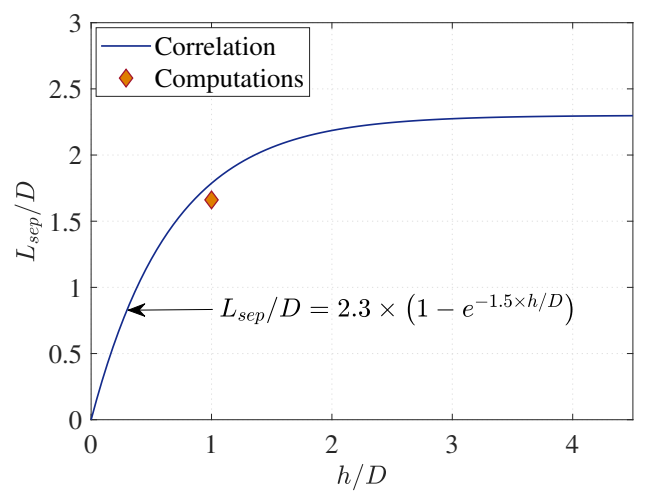

(b)

Figure 17: Comparison of computational results for cylindrical protuberance $(h / D=1)$ with the correlation presented by Sedney \& Kitchens $\operatorname{Jr}(1977)$ for $L_{\text {sep }}$ estimation for

(a) Mach 2.5 and (b) Mach 3.5 flow.

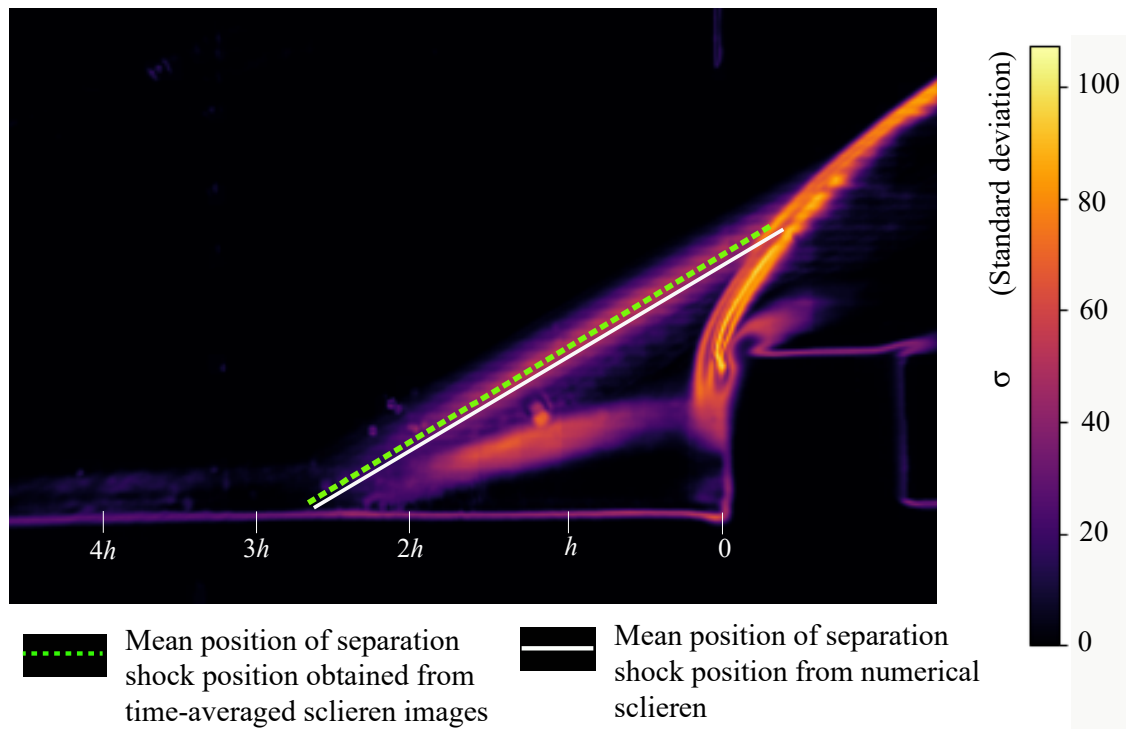

Figure 18: Standard deviation coloured, average schlieren image for $15 \mathrm{~mm}$ square-faced protuberance. The mean separation shock location from experiments and computations are marked by the dashed and the solid lines respectively.

obtained in computations is also shown, depicted by the solid line. A good match can be observed from the figure. It was observed that the mean angle of the separation shock was invariably $30^{\circ}$ for all the test cases in the experiments and computations. Although this discussion is made based on the averaged flow field, the separation shock, however, exhibits a highly unsteady motion. A brief description of the unsteady aspects is presented subsequently.

\subsection{Shock Unsteadiness}

The SBLI flow field is known to be inherently unsteady, with the separation shock exhibiting oscillations. The dynamics of the shock motion can be attributed to the upstream (incoming turbulent 


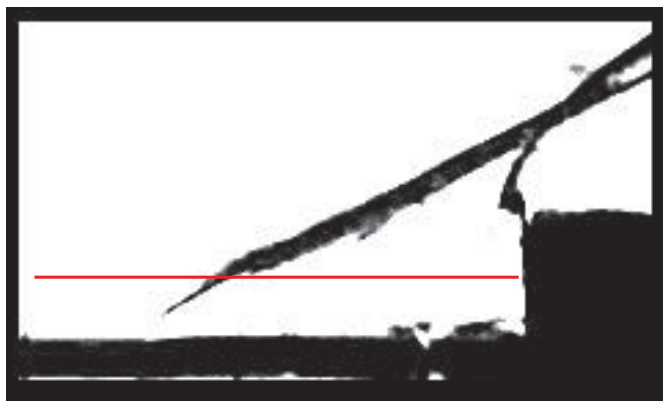

(a)

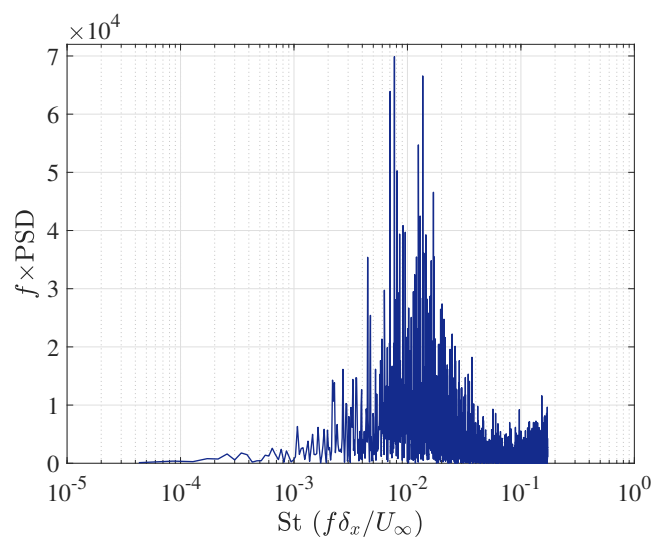

(b)

Figure 19: Spectral density estimation performed on the time-resolved schlieren images.

(a) Processed schlieren image with the line along which the pixel intensity scan was performed. (b) Pre-multiplied PSD plot with Strouhal number.

boundary layer) and downstream (within the bubble) causes. The downstream mechanisms have a greater influence on the unsteadiness of strongly separated flows, like the present interactions due to protuberances, while a mixed influence of upstream and downstream mechanism is observed for weakly separated flows (Clemens \& Narayanaswamy 2014). To identify the dominant frequencies in the flow, spectral density estimations was performed on the time-resolved schlieren snapshots. A brief discussion on the method and the results obtained is given below.

To identify the frequencies associated with the separation shock oscillations, pixel intensity of multiple schlieren snapshots was extracted along a horizontal line. Raw schlieren image (shown in figure 4) was processed by adjusting its contrast and sharpness. The raw image was also filtered to remove spots in the image caused due to markings and scratches in the glass window. This processing of the raw image was performed in order to obtain a high contrast schlieren image comprising sharp separation shock and reattachment bow shock; thus, enabling the precise capturing of the instantaneous separation shock location. Figure 19(a) shows the processed schlieren image and the horizontal line along which the intensity scan was performed. A drop in the pixel intensity marks the location of the separation shock. Upon locating the shock position for 8000 schlieren images, Fourier analysis was performed on the obtained temporal data. The pre-multiplied Power Spectral Densities (PSD) was plotted against Strouhal number as shown in figure 19(b). It can be observed that the dominant frequencies in the flow over square-faced protuberance, corresponds to a Strouhal number of the order of $10^{-2}(\sim 1000 \mathrm{~Hz})$, which is of similar orders as that for 2-dimensional normal shock induced SBLI (Murugan \& Govardhan 2016). The amplitude of shock oscillation (at shock foot) is close to $\delta_{x}$ for the case of $15 \mathrm{~mm}$ square-faced protuberance. For smaller separation lengths, such as those observed with cylinders, the amplitude was considerably lower. Thus, when $L_{\text {sep }}$ is mentioned in subsequent discussions, it shall be understood as the time-averaged value of the separation length which oscillates with an amplitude of $15-20 \%$ of the mean (i.e. $L_{\text {sep }}$ ).

\section{Results and discussions}

Geometric parameters of the protuberance, such as shape, diameter, and height, govern the length scales of the SBLI. The pressure distribution, locus of separation and reattachment, 
and primary vortex structure are thus dependent on the geometry of protuberance causing the interaction, along with the incoming boundary layer and freestream conditions. A detailed examination of the flow features and scales has been carried out using the experimental and computational data.

Although the SBLI due to protuberances is 3-dimensional, interestingly, the flow at the spanwise centre plane and its neighbourhood exhibits certain features which are similar to that of 2dimensional SBLI. This is apparent in the survey of wall static pressure along centreline, as may be observed for the cases of square-faced and cylindrical protuberances (both of $15 \mathrm{~mm}$ height and $15 \mathrm{~mm}$ width/diameter) shown in figure 15(a) and figure 16(a), respectively. Upstream of the onset of separation, there is a gradual thickening of the boundary layer due to the phenomena of upstream influence through the subsonic portion of the flow inside the boundary layer. The pressure thus starts rising a little upstream of the separation, when the boundary layer starts to thicken; the point from which the pressure starts to rise is the point of onset of the interaction (SBLI). The pressure continues to rise for a small distance after separation, and reaches a pressure plateau inside the separation bubble. Towards reattachment, the pressure starts to rise again sharply, reaching a peak value at reattachment (on the protuberance surface in the 3-dimensional case). In protuberance induced 3-dimensional SBLI, additionally, there is a local minimum in pressure between the plateau pressure region and the reattachment pressure rise, which is due to the horseshoe vortex upstream of protuberance (which shall be discussed later). Apart from this local pressure minimum for 3-dimensional SBLI, the features of streamwise variation of wall pressuresuch as plateau pressure and peak pressure at reattachment-are similar for 2-dimensional and 3-dimensional SBLI (along centre line as well as its neighbourhood). The similarity is not merely qualitative. For the 2-dimensional SBLI, the pressure rise from the onset of interaction can be obtained by means of the free interaction theory (Chapman et al. 1958). Near the wall, convection is small, and thus the near wall equations constitute the balance of pressure and wall skin friction. Integration of the 2-dimensional near wall equations, along with the consideration of the pressure rise due to the thickening of the boundary layer results in the following expression for pressure distribution along the interaction (Délery \& Marvin 1986).

$$
\frac{P}{P_{\infty}}=1+\frac{0.5 \times M_{\infty} \gamma \mathcal{F} \sqrt{2 \times c_{f}}}{\left(M_{\infty}^{2}-1\right)^{0.25}}
$$

where, $P$ is the pressure value to be calculated in the free interaction zone, $c_{f}$ is the coefficient of skin friction just upstream of the onset of the interaction, and $\gamma$ is the ratio of specific heats for the fluid. For obtaining the plateau pressure value, $\mathcal{F}$ equals 6 for a turbulent flow. For the Mach 2.89 freestream conditions, for a 2-dimensional normal shock interacting with boundary layer (at same location as with protuberance), the plateau pressure is estimated to be $1.84 \times P_{\infty}$. Interestingly, the plateau pressure observed/measured for all protuberances are close to this estimate, as may be seen in figure 15(a) and figure 16(a). A $P_{\text {plat }} / P_{\infty}$ of $\sim 2$ was observed in present computations and experiments for all protuberances at Mach 2.89 flow conditions. A good comparison between 2dimensional estimates and 3-dimensional plateau pressures were observed at other flow conditions too. Truitt (1965) presented an empirical correlation for the pressure rise across the separation shock along the centreline, for the case of cylindrical protuberances. The correlation variables were based on 2-dimensional analysis. The pressure rise due to separation was only correlated with the flow conditions upstream of interaction and were found to be independent of the cylinder height or diameter. This also means that the separation shock angle must be invariably the same for all protuberances for given upstream conditions, which was observed from the time resolved schlieren images and the computations performed in the present study. The mean separation shock from the schlieren images and from computations were shown in figure 18 for the case of squarefaced protuberance at Mach 2.89 flow, and the shock angle was observed to be $30^{\circ}$. The same 


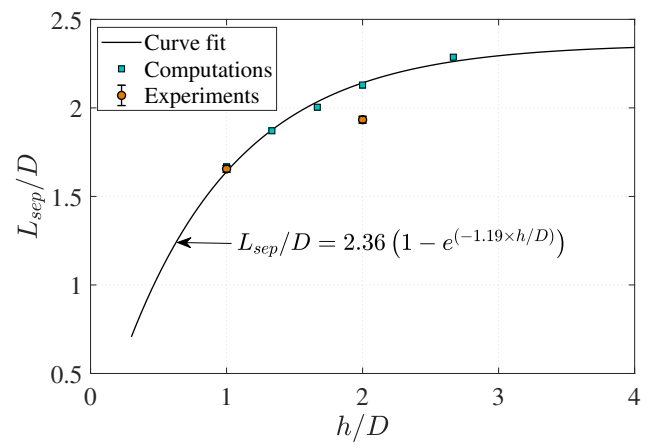

(a)

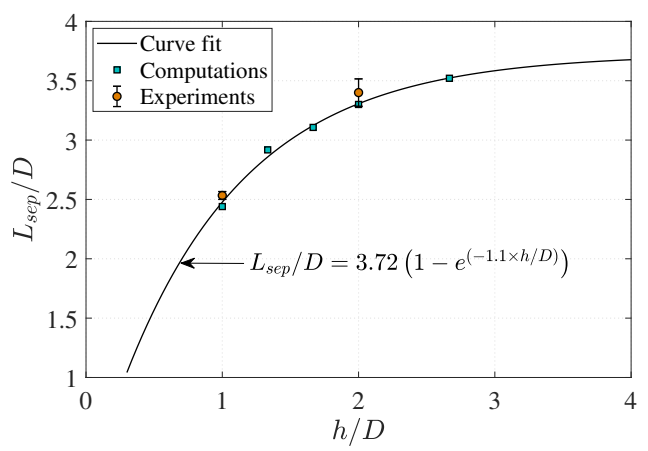

(b)

Figure 20: $L_{\text {sep }} / D$ variation with $h / D$ obtained in computations along with a comparison with the experimental measurements for (a) cylindrical protuberances and

(b) rectangular-faced protuberances.

separation shock angle was observed for other protuberances too at Mach 2.89. The flow features of 3-dimensional interactions on the centreline are thus largely similar to that of 2-dimensional interactions. The differences, particularly the local minimum after the plateau pressure, however, occur due to the presence of a horseshoe vortex for the protuberances.

Though the plateau pressures and shock angles are independent of protuberance geometry at given upstream conditions and can be predicted based on 2-dimensional considerations, the separation length is observed to be dependent on the protuberance geometry (both shape and dimensions). From the oil flow visualizations at Mach 2.89, a mean $L_{\text {sep }} / h$ for $15 \mathrm{~mm}$ height and $15 \mathrm{~mm}$ diameter cylinder was measured to be 1.67 , while the same for $15 \mathrm{~mm}$ square-faced protuberance case was 2.53 . These separation lengths increases and attain an asymptotic value as the height of the protuberance (of given shape and width) is increased. Figure 20 shows the separation length variation with the height, for cylindrical and rectangular-faced protuberances at Mach number of 2.89. Both the lengths have been normalized with the diameter/width of the protuberance $(D)$. A correlation function similar to Sedney \& Kitchens Jr (1977) was used to fit a curve to our computational data. For the cylindrical cases, asymptotic $L_{\text {sep }} / D$ was obtained to be 2.36, while for the rectangular-faced protuberances it was 3.72. Therefore, a significant difference in the separation lengths corresponding to rectangular-faced and cylindrical protuberances of same width and diameter is noted. These differences in the separation length scales, caused due to the geometry of the protuberance, lead to changes in the shock interactions and the flow features associated with them.

A constant separation shock angle and a significantly different separation length upon a change in the geometry of the protuberance results in differences in the interaction between the separation and the reattachment bow shocks. In section 2.1.1, while reflecting on Figure 4, it was mentioned that two qualitatively different types of interactions (Edney 1968) are observed between the cases of $30 \mathrm{~mm}$ height and $15 \mathrm{~mm}$ diameter cylindrical protuberance and square-faced protuberance. For the case of circular cylinders, the separation lengths are usually small, compared to rectangularfaced protuberances of comparable width. Thus, the separation shock interacts with the strong portion of the reattachment bow shock (almost normal shock), resulting in Edney's Type IV interaction. This interaction results in a supersonic jet forming due to slip streams at the vicinity of the interaction, and the impingement of the supersonic jet on the protuberance results in local peak surface pressure and heating rates above the location of shear layer reattachment. In all cylindrical and elliptic protuberances, for all freestream conditions, this was the type of the 
interaction which was observed. In the case of the rectangular-faced protuberance of $15 \mathrm{~mm}$ width and $15 \mathrm{~mm}$ height, the separation angle is the same as with any other protuberance at same freestream conditions; but when compared to the cylindrical protuberance of same diameter and height, the square-faced protuberance has larger separation length. This results in the interaction of the separation shock with a relatively weak portion of the reattachment bow shock, well above the protuberance. Type VI interaction was thus observed as shown in Figure 4(b). As the the height of the rectangular protuberance is increased, the interaction changes from Type VI to Type $\mathrm{V}$ or Type IV (depending on the height). For $15 \mathrm{~mm}$ wide rectangular protuberances, beyond a height of $25 \mathrm{~mm}$, the interaction was Type IV, occurring right in front of the protuberance. Irrespective of the type of the interaction, the patterns observed in the separated flow qualitatively remains the same, with regard to surface pressure distribution, shear layer and horseshoe vortex.

As can be observed from the above-mentioned findings, a good resemblance of the centreline flow properties with that for 2-dimensional interaction is observed, although the interaction due to protuberances is 3-dimensional. In the planes other than the spanwise centred plane, 3dimensional effects on the flow field can be observed. Firstly, considering the surface pressure profile, as we move to planes increasingly away from the centre plane, the plateau pressure keeps decreasing, as is evident from the figure 16. Moreover, the final pressure rise near the reattachment location does not occur at other planes. As a result, the pressure continues to decrease after the dip in the pressure due to the horseshoe vortex, as can be seen in figure 16(c). These differences in the pressure profiles for other planes and the spanwise centred plane are not observed for 2-dimensional interactions. Contours of pressure on the base plate are shown in figures 21(a) and 21(b) for cylindrical protuberance of height and diameter of $15 \mathrm{~mm}$, and squarefaced protuberance respectively. Based on the levels of pressure, various zones can be identified in the interaction. Figures 21(c) and (d) highlight the different pressure zones for cylindrical and square-faced protuberances. Significant differences in the extent of plateau pressure zone as well as of other zones can be observed for the two cases. A larger mass of nearly stagnant fluid (high pressure zone) in front of the square-faced protuberance (region I) can also be observed from the contour of the surface pressure. These differences in the extent of different pressure zones for different geometries of the protuberance manifest in significantly different separation length scales.

The separated flow at the centreline and its neighborhood reattaches on the protuberance surface, resulting in peak pressures at the location of reattachment. Further, the reattaching streamlines at the centreline encounter normal shock before reattachment, resulting in near stagnation of the reattaching flow. The shape of the protuberance plays a determining role in the spanwise extent of this near-stagnant reattached portion of the gas and its subsequent flow direction. The skin friction forces acting on the reattached fluid close to the protuberance surface, are strongly influenced by the shape of the protuberance. Figure 22 shows a contour of skin friction coefficient on the front face of the cylindrical (15 mm height; $15 \mathrm{~mm}$ diameter) and square-faced protuberances. Skin friction lines and vectors have also been shown in the same figure to identify the reattachment location and aid in understanding the flow direction close to the protuberance surface. Difference in the spanwise extent of the reattachment zone, having near zero skin friction coefficient, can be observed from the figure for the two protuberances. The reattachment zone for the square-faced protuberance extends to a significant proportion of its width, while it is mostly concentrated close to the centreline for the cylindrical case. This varied extents of the reattachment zone on the surface due to the shape of the protuberance, provide a qualitative measure of the obstruction offered by the protuberance to the separated flow. A considerable increase in the skin friction occurs in azimuthal direction for the cylindrical protuberance, while it maintains low values for a significant spanwise distance for the square-faced protuberance. This difference in the skin friction forces on the front face of the protuberance results in different flow directions close to the surface for the two cases, as shown in the figure. The reattached flow in the 


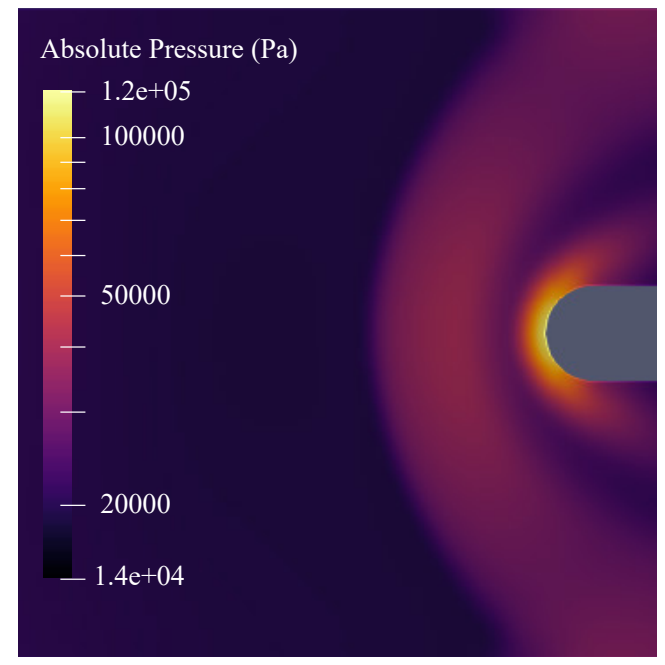

(a)

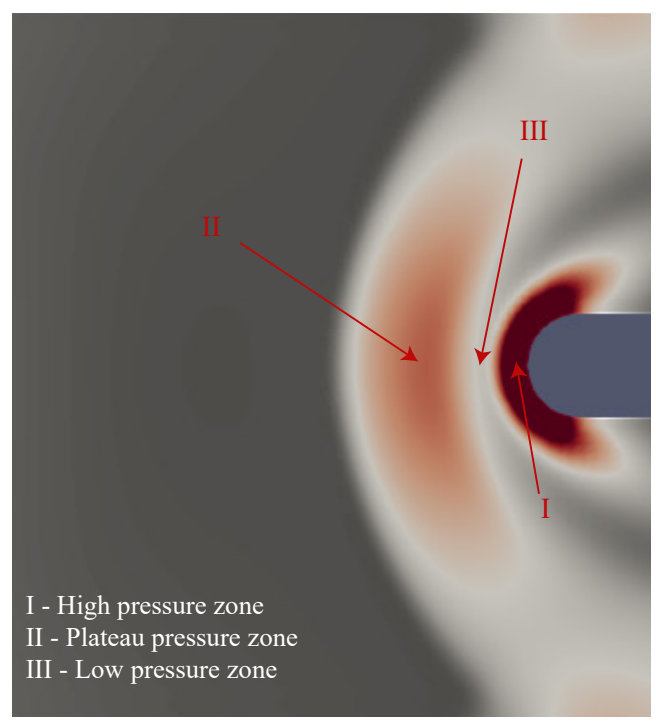

(c)

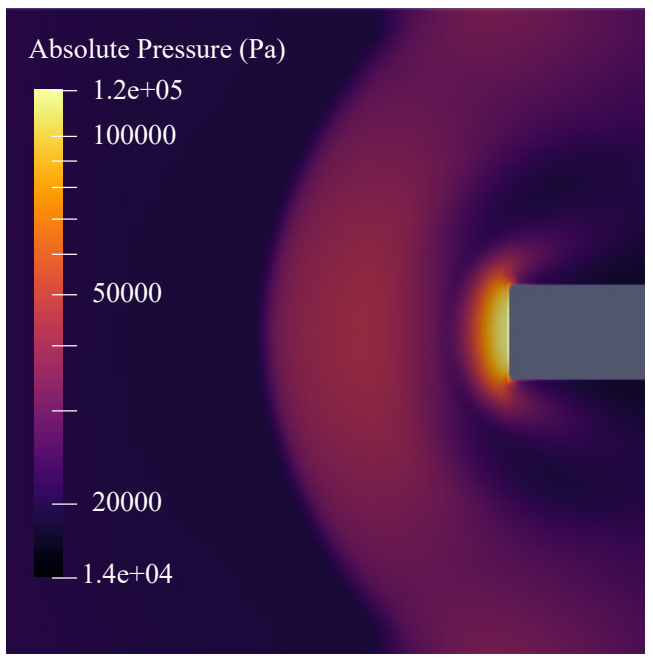

(b)

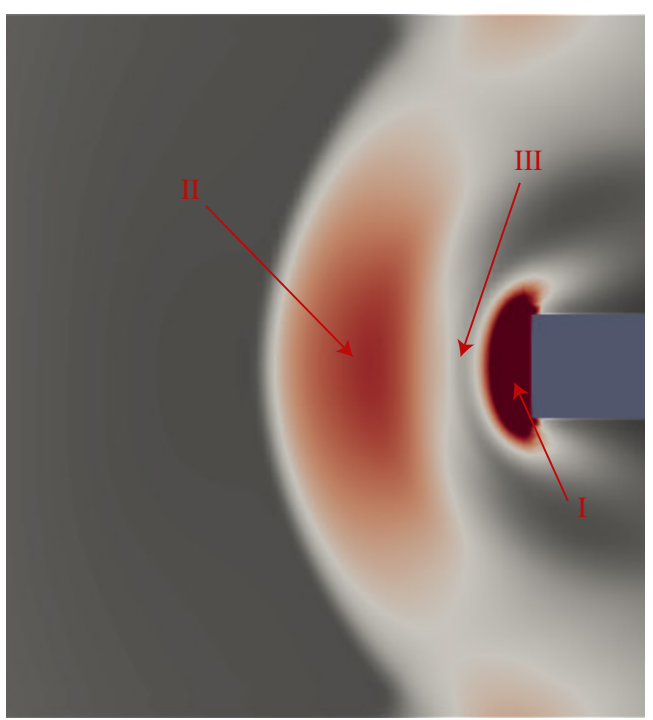

(d)

Figure 21: Pressure contour on the base plate for (a) $15 \mathrm{~mm}$ diameter and $15 \mathrm{~mm}$ height cylindrical protuberance and (b) square-faced protuberance of $15 \mathrm{~mm}$ edge. Different pressure zones for (c) the cylindrical and (d) the square-faced protuberance.

centreline flows straight down towards the base, while the flow away from the centre goes towards the sides of the protuberance. The curvature of the front face of the protuberance influences the sideways movement of the near stagnant flow. This results in quicker relieving of pressure in the spanwise direction for cylindrical protuberances compared to the square-faced protuberance, where downward movement of the reattached flow occurs for a wider extent, as can be observed from the surface streakline pattern in the figure 22. This reattached flow in the centreline gains considerable velocity while moving towards the base plate and subsequently travels upstream. The surface streaklines of the reattached flow travelling upstream in the recirculation region can be traced by oil flow visualization on the base plate. Figure 23 shows the surface streaklines for 


Skin Friction Coefficient
$8.9 \mathrm{e}-03$
$6.9 \mathrm{e}-03$
$4.9 \mathrm{e}-03$
$3.0 \mathrm{e}-03$
$9.8 \mathrm{e}-04$

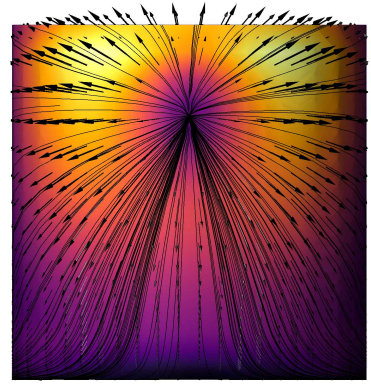

(a)

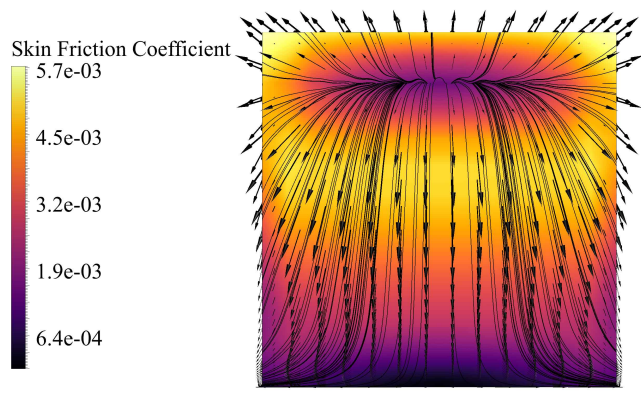

(b)

Figure 22: Surface streaklines along with vectors and contour of skin friction coefficient on the surface of (a) cylindrical and (b) square-faced protuberance.

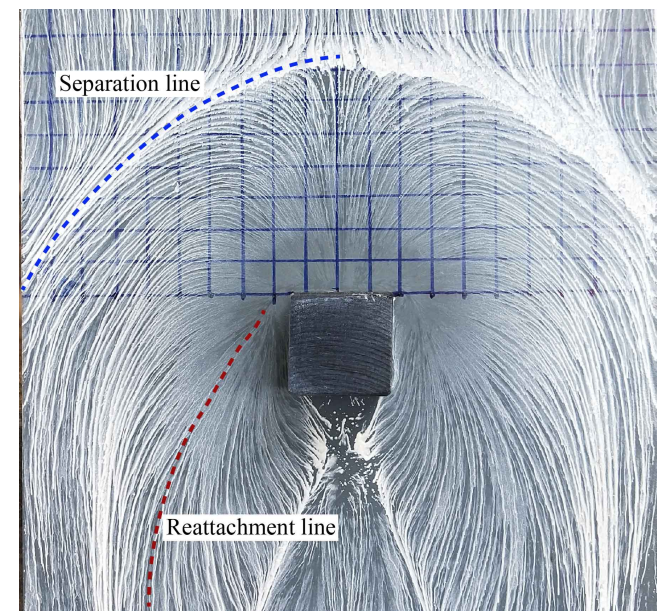

Figure 23: Oil flow visualization showing the separation and the reattachment locations on the base plate for $15 \mathrm{~mm}$ square-faced protuberance.

the case of square-faced protuberance. Unlike the oil distribution for the measurement of $L_{\text {sep }}$, a denser distribution of the oil mixture was used in order to reveal finer flow details in the separation zone, although it results in smudged separation line. The reattachment line on the base plate has also been marked, which is discussed later. Occurrence of a secondary separation or corner vortex has been reported in the literature (Hung \& Buning 1985; Ozawa \& Laurence 2018; Lakshmanan $\&$ Tiwari 1994). The corner vortex was difficult to identify in the present study. The upstream travel and subsequent turning of the reattached flow in the downstream direction can be observed from the oil flow pattern shown in the figure. From the computational results, the surface streakline pattern on the base plate for cylindrical and square-faced protuberances is shown in figure 24, coloured with skin friction coefficient magnitude. For the case of square-faced protuberance, more number of reattached streamlines travel in the upstream direction after their downward movement, and thus lesser relieving on the protuberance surface. The surface streaklines thus travel upstream to larger distances for the square-faced protuberance before turning downstream. These differences, in general, results in larger separation lengths for flat-faced protuberances as compared to curved protuberances of same width and height.

Due to the 3-dimensional relieving effect in the recirculation zone, there are some pockets of supersonic flow in the recirculation region even at the mid-span near the protuberance as 


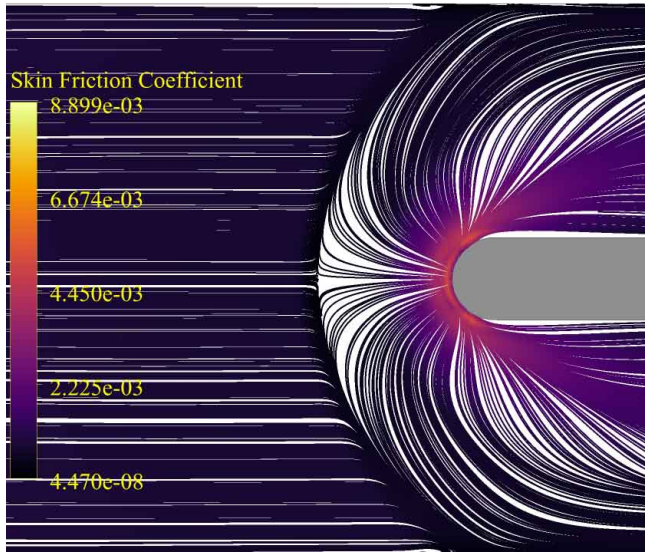

(a)

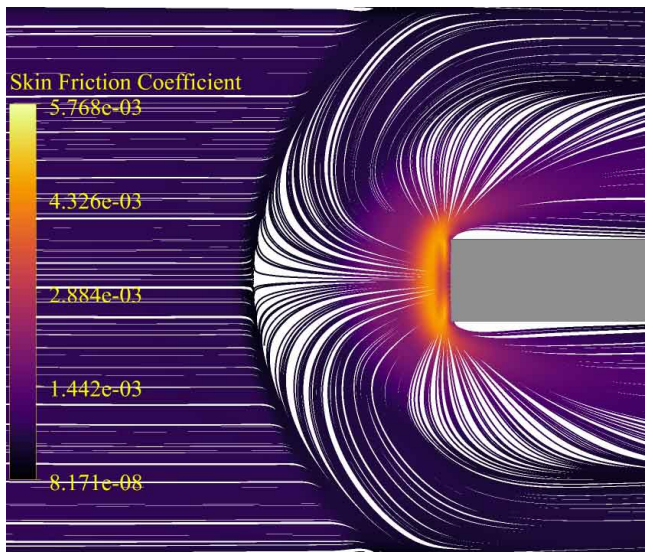

(b)

Figure 24: Surface streaklines coloured with skin friction coefficient on the base plate for

(a) $15 \mathrm{~mm}$ diameter and $15 \mathrm{~mm}$ height cylindrical protuberance and (b) square-faced protuberance.

well as the plate walls. Figure 25 shows the contour of Mach number for $M>1$ depicting the supersonic zones in the spanwise centred plane for the square-faced protuberance. To aid in the understanding of shock structures, numerical schlieren is present in the background of the figure. The subsonic flow behind the bow shock starts accelerating towards the base plate and attains supersonic velocities. It attains a Mach number of 1.2 close to the protuberance surface after which a normal shock terminates this supersonic flow. The reattached fluid then turns near the corner and again reaches supersonic velocities with Mach number of 1.4 close to the base plate above the location where a local dip in surface pressure was observed between the plateau and peak pressure (marked as region III in figure 21). Subsequently, the reattached fluid gets entrained in the primary horseshoe vortex and moves downstream. Unlike a 2-dimensional interaction, where a closed primary vortex is observed, fluid particles entrained in the vortex formed in 3-dimensional interactions, move in helical fashion due to the availability of an extra dimension along the span. Streamlines that reattach on the protuberance front face mark the reattachment location on the surface of the protuberance, as shown in figure 22. All the streamlines above this reattaching streamline go in the direction of the shoulder of the body. Streamlines below the reattaching streamline get entrained in the primary vortex and travel downstream in helical motion.

In order to analyze the motion of the fluid particles in the recirculation zone, streamlines originating from four different wall-normal distances upstream of the interaction zone and in the centreline plane are traced for the square-faced protuberance, as shown in figure 26 . The streamlines originate at wall-normal distances $(y / h)$ of 0.048 (close to the wall), $0.21,0.48$ (edge of the boundary layer), and 0.75 (in the outer flow). All the streamlines have different initial stagnation pressures as they are originating from different regions of the flow. The stagnation pressure of the streamlines continuously reduces as they flow downstream in the interaction zone. The reduction in the stagnation pressure for the streamline originating at $y / h=0.48$ is a result of the fluid particle passing through the separation shock and the reattachment bow shock. Near the front face of the protuberance, the fluid particle becomes nearly stagnant and marks the location of reattachment on the protuberance. Streamline at $y / h=0.21$, having a lower stagnation pressure, after passing through the two shocks starts moving towards the base plate. Streamlines at comparable wall-normal distances may achieve supersonic speed, which is terminated by the normal shock present on the protuberance surface, as discussed earlier, 


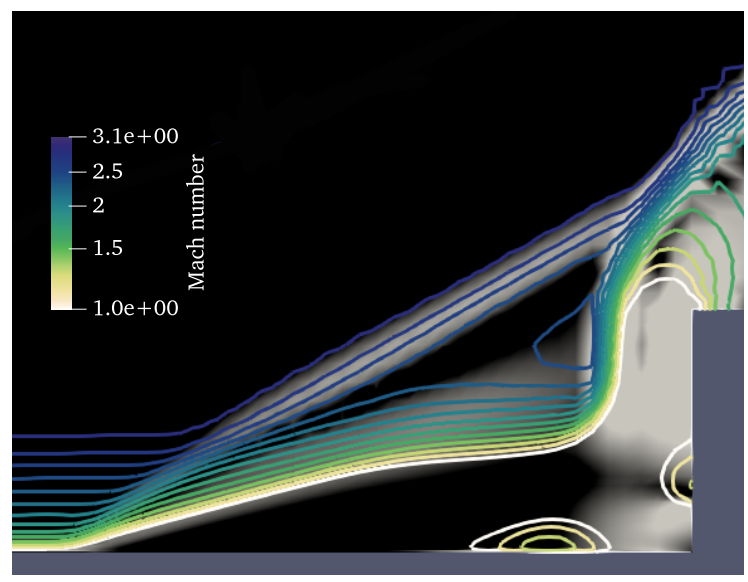

Figure 25: Contour of Mach number for $M>1$ for $15 \mathrm{~mm}$ edge square-faced protuberance with numerical schlieren in the background.

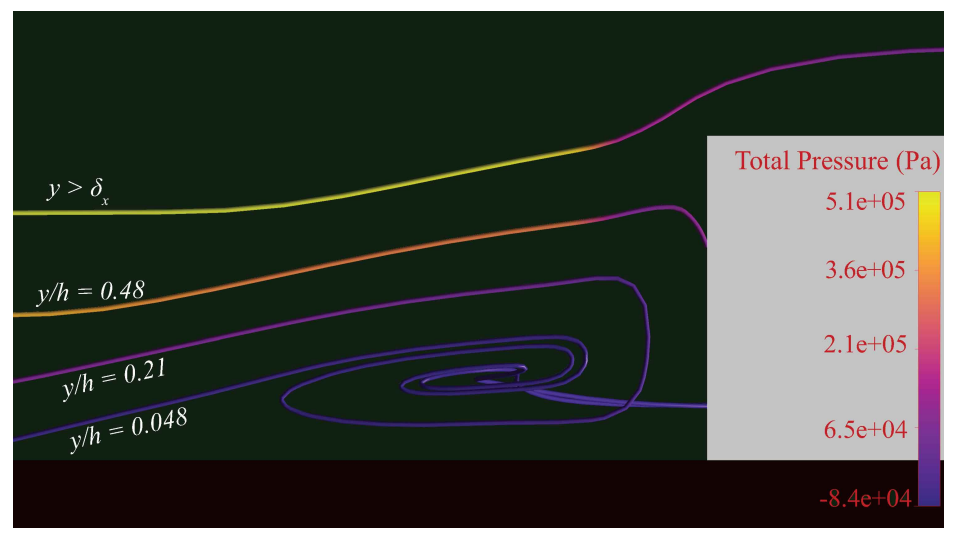

Figure 26: Variation of stagnation pressure along the streamlines originating at different wall-normal distances for the square-faced protuberance.

thus reducing the stagnation pressure further. These streamlines exhibit vortical motion and move towards the core of the vortex. As the streamlines travel through the separated flow, the stagnation pressure continues to decrease and becomes comparable to that for low energy streamline originating close to the wall (at $y / h=0.048$ ), which directly gets entrained in the vortex core. It is observed that the streamlines originating at the wall-normal distances less than the boundary layer thickness, get entrained in the vortex and move towards its core and in the process, their stagnation pressure reduces continuously. While, the streamlines originating at the edge of the boundary layer $(y / h=0.48)$ reattaches at the protuberance surface. The streamlines originating at $y>\delta_{x}$ (for example $y / h=0.75$ ) turn towards the shoulder of the protuberance.

As seen from the previous discussion, the fluid in the recirculation zone is characterized by horseshoe vortex. Dynamics of strong SBLI due to 2-dimensional and 3-dimensional obstructions are highly influenced by the dynamics of the recirculation zone and the location of the vortex core. As the flow field in front of the protuberance depends on its geometry, the location and the profile of the horseshoe vortex is expected to be different for the cylindrical and square-faced protuberances of similar dimensions. In order to visualize the horseshoe vortex, $\lambda_{2}$-criterion was employed using the computational results. $\lambda_{2}$ is the second largest eigenvalue of $\Omega^{2}+\boldsymbol{S}^{2}$ where, $\Omega$ is the vorticity tensor and $\boldsymbol{S}$ is the strain rate tensor which are anti-symmetric and symmetric 
components of gradient of velocity $(\nabla u)$, respectively. A negative value of $\lambda_{2}$ is taken to identify the vortex core in the computational domain. While an accurate criterion for vortex identification is still debated, a recent study by Yao \& Hussain (2018) suggests that $\lambda_{2}$-criterion identifies vortex core better for compressible flows with $M<5$. Figures 27(a) and 27(b) shows the vortex core for $15 \mathrm{~mm}$ height cylinder and square-faced protuberances, respectively. As can be observed for the square-faced protuberance case, the vortex core breaks in front of the protuberance. It has been reported in the literature (Kolár 2007) that the vortex core may be discontinuous at the location where there is a strong axial stretching. This usually happens with both $\lambda_{2}$ and $Q$ vortex identification criteria. The streamlines originating at different wall-normal distances, as described earlier, are also shown in the figure. Entrainment of the streamlines in the vortex and subsequent movement in the downstream direction can be observed from the figure. Figures 27(c) and 27(d) shows the plan view of the horseshoe vortex and the motion of the incoming streamlines after getting entrained in the vortex. The differences in the horseshoe vortex for the cylindrical and square-faced protuberances results in variations in the interaction length scales. The dependence of mean $L_{\text {sep }}$ on the core of the vortex for various geometries is examined in section 4.

In the planes away from the centreline, the reattachment and subsequent direction of the flow is different from that observed for the centreline flow and its close neighborhood, which was previously shown in figures 26 and 27. The streamlines originating at different wall-normal distances in the planes away from the centreline along with surface streakline patterns on the base plate and the protuberance surface are shown in figure 28. Entrainment of the streamlines originating close to the base plate in the horseshoe vortex is not observed, unlike for the streamlines originating in planes closer to the spanwise centre. The streamlines reattaching on the protuberance surface move towards the protuberance corner and subsequently, in the downstream direction. This high pressure reattached flow is demarcated from the rest of the flow by the reattachment line on the base plate which was shown in figure 23. This can also be observed near the corner of the protuberance from the surface streakline pattern shown in the figure 24 .

\section{Scaling law}

For nominally 2-dimensional interactions, a theoretical treatment of the SBLI and separation is enabled by the free interaction theory (Chapman et al. 1958) and the triple-deck formulation of boundary layer (Stewartson \& Williams 1969). The theoretical prediction of the pressure rise due to separation based on the free interaction theory, and the good comparison of measured centreline plateau pressure for the case of 3-dimensional protuberances with the estimates from the theory was discussed in section 3. Scaling laws for separation length have been obtained by integrating the equations based on triple deck formulation by Burggraf (1975), Rizzetta et al. (1978) and Davis $\&$ Sturtevant (2000) for laminar SBLI. Based on the data obtained from numerical simulations of 2-dimensional laminar SBLI, Katzer (1989) presented the following correlation:

$$
\frac{L_{s e p} M_{\infty}^{3}}{\delta_{i}^{*}\left(R e_{x i} / C\right)^{0.5}}=4.4 \frac{P_{s}-P_{i n c}}{P_{\infty}}
$$

where, $P_{i n c}$ is the incipient pressure. $R e_{x i}$ and $\delta_{i}^{*}$ are the Reynolds number and the displacement thickness at the impingement location, respectively. $C$ is the Chapman-Rubesin constant.

Similar correlations were also obtained based on experiments for laminar SBLI (Davis \& Sturtevant 2000; Sriram \& Jagadeesh 2015; Sriram et al. 2016). While the variables in the correlations based on experimental and numerical data are the same or a modified form of the variables in the theoretically obtained scaling laws, notably the pressure term (the term in the right hand side of equation 4.1) is linear in the correlations, while the theory predicts a non-linear pressure term with an exponent of 1.5. Although the scaling law is for laminar separation bubble, 


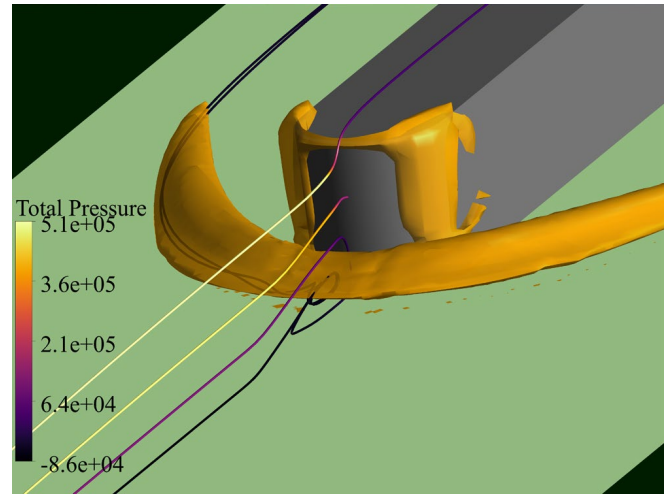

(a)

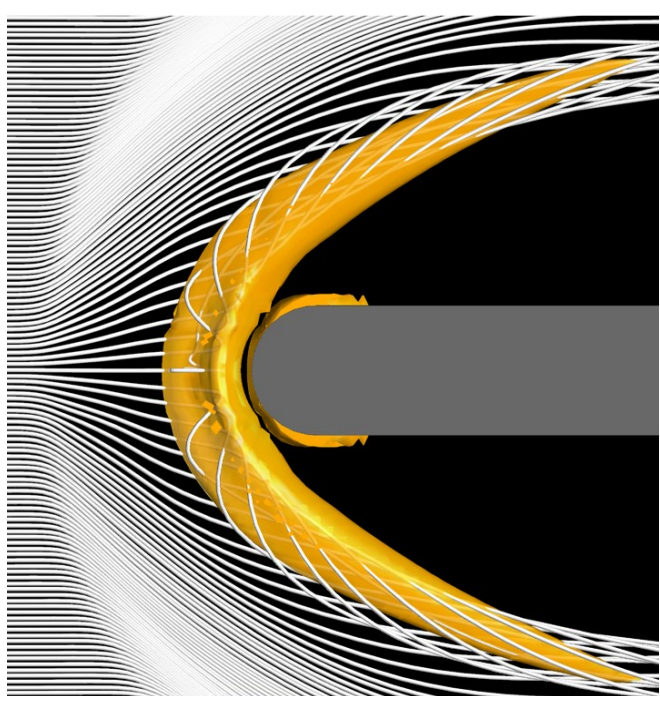

(c)

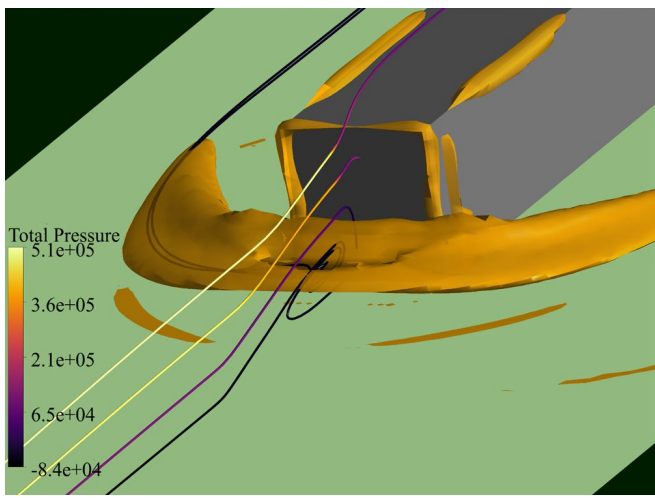

(b)

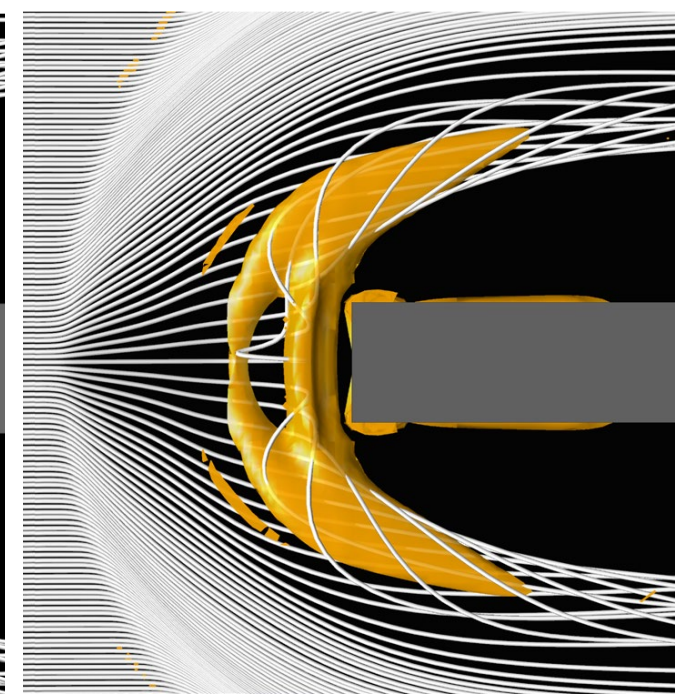

(d)

Figure 27: Identification of horseshoe vortex and flow of streamlines originating at different wall-normal distances in the spanwise centred plane for (a) cylindrical (15 mm height and $15 \mathrm{~mm}$ diameter) and (b) square-faced protuberance. Top view showing the entrainment of streamlines around the vortex core and their helical motion for (a) cylindrical (15 $\mathrm{mm}$ height and $15 \mathrm{~mm}$ diameter) and (b) square-faced protuberance.

the trends with Mach number and and shock strength (pressure term) predicted by the scaling law are also expected for turbulent SBLI. The separation length must increase with the shock strength, and must decrease with Mach number due to relatively smaller subsonic portion in the boundary layer. The relation with Reynolds number is based on the inverse relation between the separation length and the skin friction: the larger the skin friction, the more resistant the boundary layer to adverse pressure gradient, and thus, smaller the separation length. The exponent of 0.5 for Reynolds number in the correlations is because the studies are concerning laminar SBLI. For turbulent SBLI, the exponent should be 1/5, signifying lesser influence of Reynolds number.

Although the 3-dimensional SBLI are too complicated for theoretical treatment, as discussed before, the pressure rise at separation along the centreline could be obtained from a 2-dimensional analysis of normal shock boundary layer interaction. This meant that for given upstream 


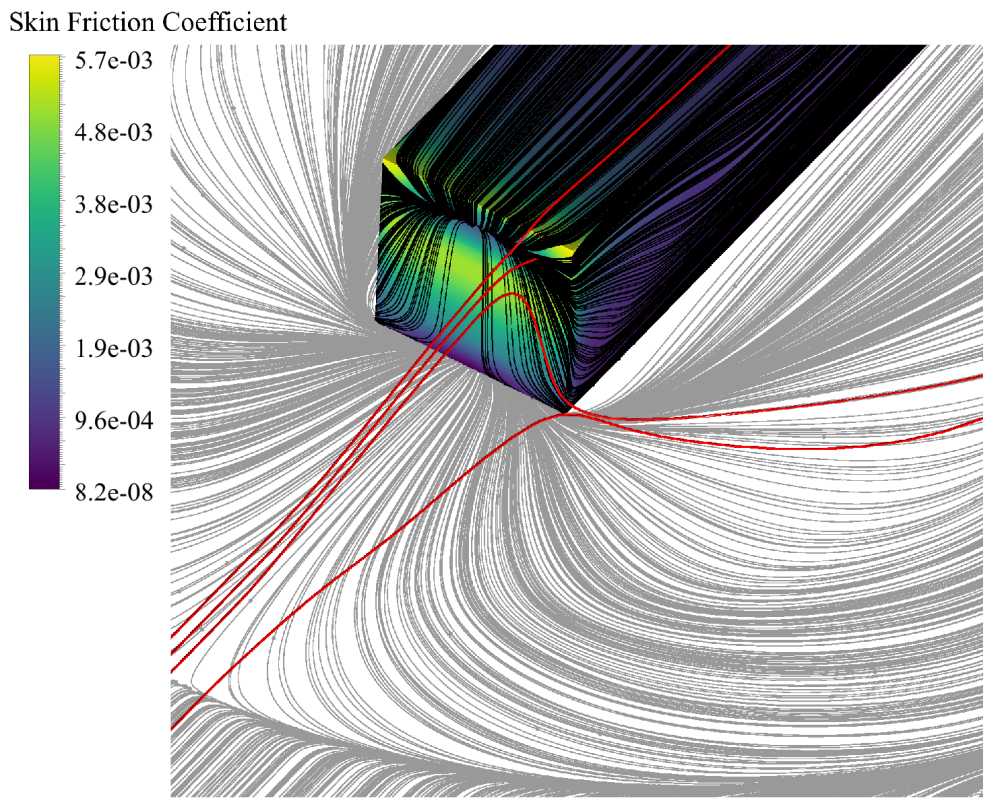

Figure 28: Flow of streamlines originating at different wall-normal distances in a plane away from the spanwise-centred plane. Surface streaklines are shown on the square-faced protuberance surface and the base plate. Contour of skin friction coefficient on the protuberance surface is also shown.

conditions, the plateau pressure was independent of the geometry of the protuberance, which was also observed experimentally. However, the separation length was geometry dependent. Attempts to obtain correlations for separation length (or equivalently the length of the plateau region) similar to those in 2-dimensional SBLI, even at the centreline, are thus complicated by the geometric considerations of 3-dimensional interactions. A careful examination of the dependence on geometry is firstly required.

For the case of protuberances, the geometrical parameters include height, diameter/width, and shape of the protuberance. Apart from these geometrical parameters, the separation and the overall flow field also depend on incoming flow parameters such as Mach number and Reynolds number. For a given geometric shape, from dimensional considerations, the dependence of separation length (non-dimensionalized by $\delta_{x}$ ) can be given as:

$$
\frac{L_{\text {sep }}}{\delta_{x}}=\mathcal{F}\left(\frac{h}{\delta_{x}}, \frac{D}{\delta_{x}}, R e_{x}, M_{\infty}\right)
$$

Here, $h$ is the height of the protuberance, $D$ is the width/diameter of the protuberance. $\delta_{x}$ is the boundary layer thickness. $R e_{x}$ is the Reynolds number based on distance from the leading edge. The relation between $L_{\text {sep }} / D$ and $h / D$ for fixed Mach numbers was discussed previously in section 2.3 and in section 3. The empirical correlation by Sedney \& Kitchens Jr (1977) for cylindrical protuberances at Mach numbers of 2.5 and 3.5 were shown in figure 17; the correlations based on present study for cylindrical and rectangular protuberances at Mach number of 2.89 were shown in figure 20. The separation length is observed to achieve an asymptotic value as the height of the protuberance is increased well above the boundary layer thickness. The correlations as well as the asymptotic $L_{s e p}$ values were observed to be varying with Mach number and protuberance shape. In a recent work, Lindörfer et al. (2020) presented empirical correlation, relating the separation length and the diameter of semi-infinite cylinders at Mach number of 2. Both these length scales 


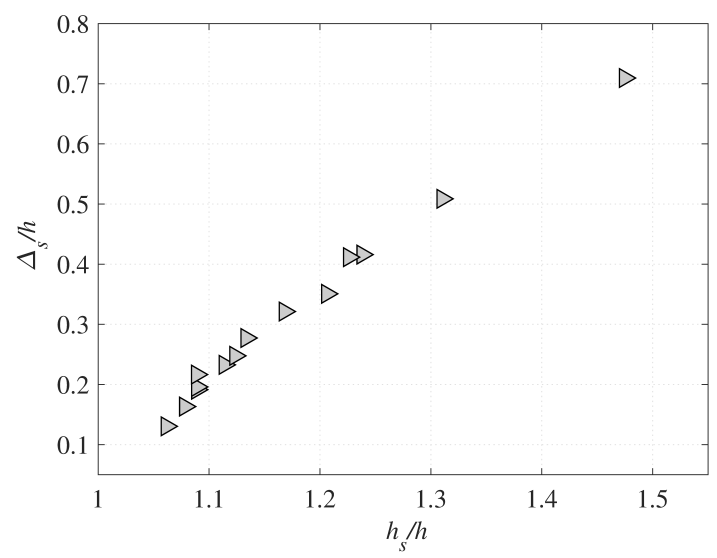

Figure 29: $\Delta_{S} / h$ variation with $h_{s} / h$ for all protuberances at Mach 2.89 flow.

were non-dimensionalized by $\delta_{x}$. It was reported that the non-dimensionalized $L_{s e p}$ varied linearly with the scaled diameters. In summary, all the above works in the literature thus attempted to relate $L_{s e p}$ with one of the non-dimensional length scale, particularly for cylindrical protuberances, for fixed freestream conditions. There are no universal scaling laws such as those for 2-dimensional SBLI (for example, equation 4.1), owing to the apparent complexities of the 3-dimensional SBLI. However, the inviscid bow shock in front of the protuberance, which is essentially the shock interacting with the boundary layer, is a common feature for protuberances of all shapes and dimensions. The dimensions related to the bow shock shall thus provide some universal scales, using which the present study attempts to seek universal scaling laws for protuberance induced SBLI.

The inviscid bow shock stand-off distance $\left(\Delta_{S}\right)$ depends on the upstream flow conditions as well as the geometry of the protuberance. The stand-off distance is thus an important parameter to consider. The bow shock is convex towards the upstream flow, normal to the flow at the axis (in case of protuberance under consideration, wall acts as 'mirror'; the axis is thus on the junction of the wall plane and the spanwise centre plane), reducing in angle as one moves away. The distance of the sonic point on the shock at different planes provides an important scale. It tells the length over which the angle changes by a fixed amount. The shock angle at the sonic point is also close to the shock angle above which the oblique shock solutions are 'strong'; thus, the sonic point distance aids in identifying the strong shock region downstream of the inviscid shock. The wall-normal distance from the base plate to the sonic point on the inviscid shock in the centreline plane is measured as $h_{s}$ while, $w_{s}$ is measured to be the spanwise distance from the centreline plane to the sonic point on the inviscid shock at the plane of the base plate. It is however observed that for a particular Mach number, $h_{s}$ and $\Delta_{s}$ are linearly related for all protuberance geometries, as shown in figure 29. Further, the role of the vertical dimensions could be significant if the bow shock changes its angle rapidly near wall, thus imposing a varying pressure gradient along the boundary layer. Such a case is expected only when the protuberances are considerably smaller than the boundary layer. For protuberances comparable to the boundary layer thickness or larger than that, the inviscid shock is nearly normal to the plane of the base plate till near the top edge of the protuberance. Therefore, $h_{s}$ is not an independent parameter for the case of protuberances.

The extent of the strong shock region of the inviscid shock is nearly described by $w_{s}$. A good correlation between $L_{s e p}$ and the parameters from the strong inviscid shock portion is observed. Figure 30 shows a 3-dimensional plot of $L_{s e p}$ variation with $w_{s}$ and $\Delta_{s}$, non-dimensionalized by $h$, at Mach number of 2.89. A plane has been fitted with the data, having a $R^{2}$ value of 0.99 . 


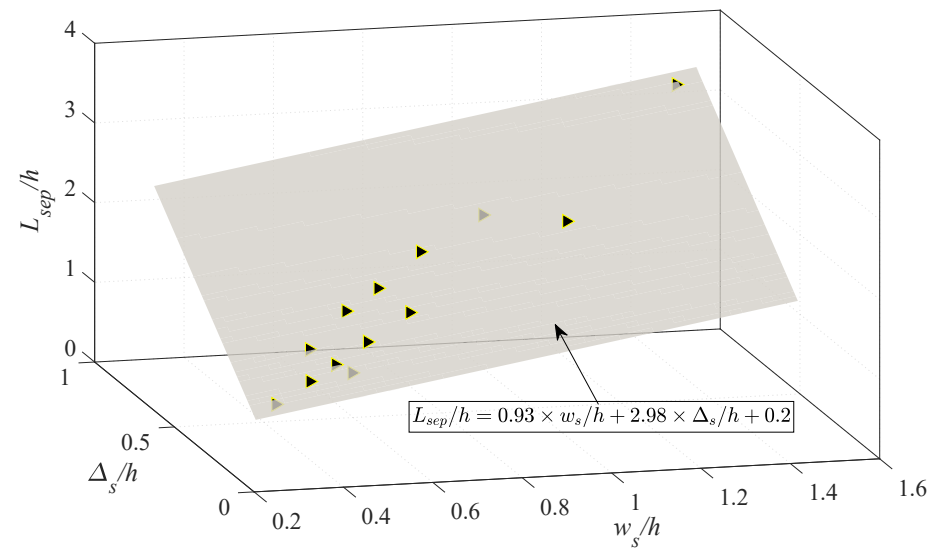

Figure 30: A 3-dimensional plot of $L_{s e p} / h, w_{s} / h$, and $\Delta_{S} / h$ for all the geometries at Mach 2.89 flow. A plane is fitted to the data.

The points for all the protuberance geometries fall on the same plane; however, the data points for other Mach numbers do not fall on this plane; they might fit in other planes corresponding to their Mach number. It can also be observed from the figure that the data points fall in two straight lines in the plane fit. The points falling in a straight line are for a particular shape of the protuberance. Therefore, it was found that for a given shape of the protuberance, $w_{s}$ and $\Delta_{s}$ were linearly related. A larger extent of the strong shock region $\left(w_{s}\right)$ signifies more spanwise extent of streamlines which are compressed to the pressure comparable to the peak pressure at reattachment in the spanwise centred plane $\left(P_{\text {peak }}\right)$. Figure 31 shows the variation of static pressure $P$ and total pressure $P_{\circ}$ along streamlines near the reattachment location for cylinder and square-faced protuberance (both $15 \mathrm{~mm}$ height and $15 \mathrm{~mm}$ diameter/width). On comparing the pressure for the two different geometries, the difference in the number of streamlines achieving pressure comparable to the peak pressure can be observed. In the figure, a red colored region has also been marked to show the extent of high pressure fluid, with pressure reaching $80 \%$ of $P_{\text {peak }}$ or more. A significant difference in the spanwise extent of this high pressure region can be seen for the two cases. Higher spanwise distance of the streamlines at freestream, reaching peak pressure or comparable value is indicative of lesser relieving of the pressure due to the shape of the protuberance. Based on these considerations, the separation length (or the length of the plateau region) in the centreline is expected to be influenced by both the peak pressure (as with the 2-dimensional cases) as well as the relieving of the streamlines away from the centreline, particularly in the strong shock portion. This relieving of the flow in the strong shock portion after the inviscid shock can be characterized by the gradient of pressure along the inviscid bow shock.

Let $P_{R}$ be the pressure at any point immediately after the inviscid bow shock (it is thus a function of the position on the bow shock). The radius of curvature of the inviscid shock at the nose $\left(R_{N}\right)$ can be related to the second derivative of $P_{R}$ along the inviscid shock at the nose. The relation between the pressure gradient and the geometric scale of the inviscid shock is derived below. Figure 32 defines the parameters used and the sign convention followed while deriving the relation. $\alpha$ and $\beta$ are the angles, tangent at a point on the inviscid shock make with the positive $x$-axis and the flow direction respectively; ' $\mathrm{s}$ ' is the distance measured along the shock, with $\mathrm{s}=0$ at nose. Anti-clockwise sense of rotation is taken positive. Radius of curvature $\left(R_{c}\right)$ at a point on 


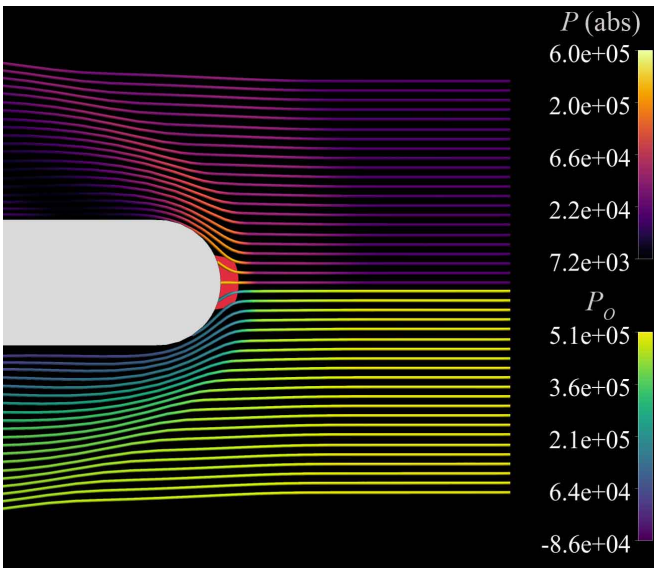

(a)

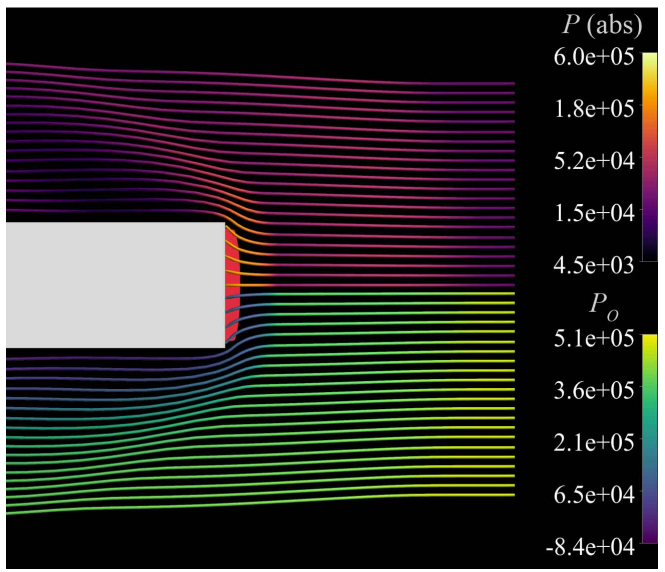

(b)

Figure 31: Streamlines in the vicinity of reattachment, coloured with absolute pressure and total pressure for (a) $15 \mathrm{~mm}$ height and $15 \mathrm{~mm}$ diameter cylinder and (b) square-faced protuberance. A red coloured region has been marked with pressure values reaching $80 \%$ of $P_{\text {peak }}$ or more.

the inviscid shock is given by,

$$
R_{c}=\frac{d s}{d \alpha}
$$

Re-writing the second derivative of $P_{R}$ as:

$$
\begin{gathered}
\frac{d^{2} P_{R}}{d s^{2}}=\frac{d}{d \alpha}\left(\frac{d P_{R}}{d s}\right)\left(\frac{d \alpha}{d s}\right)=\frac{1}{R_{c}} \frac{d}{d \alpha}\left(\frac{d P_{R}}{d s}\right) \\
\frac{d^{2} P_{R}}{d s^{2}}=\frac{1}{R_{c}} \frac{d}{d \alpha}\left(\frac{1}{R_{c}} \frac{d P_{R}}{d \alpha}\right) \\
\frac{d^{2} P_{R}}{d s^{2}}=-\frac{1}{R_{c}^{3}} \frac{d P_{R}}{d \alpha} \frac{d R_{c}}{d \alpha}+\frac{1}{R_{c}^{2}} \frac{d^{2} P_{R}}{d \alpha^{2}}
\end{gathered}
$$

On the left side of plane of symmetry, the pressure downstream of shock using the oblique shock relations

On the left $\beta=\alpha-\pi / 2$,

$$
\frac{P_{R}}{P_{\infty}}=1+\frac{2 \gamma}{\gamma+1}\left(M_{\infty}^{2} \sin ^{2} \beta-1\right)
$$

$$
\frac{P_{R}}{P_{\infty}}=1+\frac{2 \gamma}{\gamma+1}\left(M_{\infty}^{2} \sin ^{2}(\alpha-\pi / 2)-1\right)
$$

Differentiating with respect to $\alpha$,

$$
\frac{d P_{R}}{d \alpha}=-\frac{2 \gamma}{\gamma+1} M_{\infty}^{2} P_{\infty} \sin (2 \alpha)
$$

Similarly on the right side, where $\beta=\alpha+\pi / 2,\left(d P_{R} / d \alpha\right)$ also results in (4.4). Thus,

$$
\frac{d^{2} P_{R}}{d \alpha^{2}}=-\frac{4 \gamma}{\gamma+1} M_{\infty}^{2} P_{\infty} \cos (2 \alpha)
$$




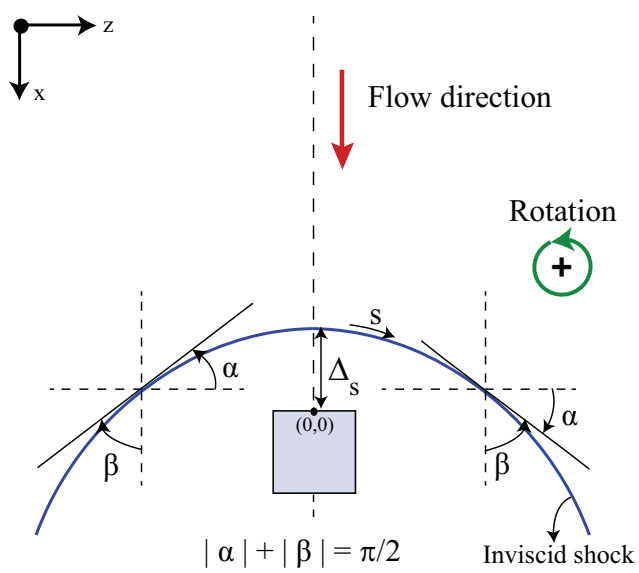

Figure 32: Schematic showing different parameters and the sign convention followed for obtaining a relationship between the second derivative of $P_{R}$ with the radius of curvature of the inviscid bow shock at the nose $\left(R_{N}\right)$.

Substituting in (4.3),

$$
\frac{d^{2} P_{R}}{d s^{2}}=+\frac{1}{R_{c}^{3}} \frac{d R_{c}}{d \alpha}\left(\frac{2 \gamma}{\gamma+1} M_{\infty}^{2} P_{\infty} \sin (2 \alpha)\right)-\frac{1}{R_{c}^{2}} \frac{4 \gamma}{\gamma+1} M_{\infty}^{2} P_{\infty} \cos (2 \alpha)
$$

At the nose of the inviscid shock, $\alpha=0$,

$$
\left(\frac{d^{2} P_{R}}{d s^{2}}\right)_{N}=-\frac{1}{R_{N}^{2}} \frac{4 \gamma}{\gamma+1} M_{\infty}^{2} P_{\infty}
$$

Normalizing with $\delta_{x}$,

$$
-\left(\frac{d^{2} P_{R}}{d s^{2}}\right)_{N} \frac{\gamma+1}{4 \gamma} \frac{\delta_{x}^{2}}{M_{\infty}^{2} P_{\infty}}=\frac{\delta_{x}^{2}}{R_{N}^{2}}
$$

The above expression (4.7) provides a direct measure of the second derivative of the pressure jump along the inviscid shock at the nose of the shock and relates it to a geometrical scale of the inviscid shock i.e. $R_{N}$. It was also observed that $R_{N}$ varies almost linearly with $w_{s}$ as shown in the figure 33. Therefore, apart from giving an estimation of the gradients of peak pressure, $R_{N}$ also represents the spanwise extent of the strong shock region of the inviscid shock. To corroborate the relation of $R_{N}$ with $w_{s}$, a comparison is drawn between the inviscid shock profiles of two different protuberances having nearly same $R_{N}$. Figure 34 compares the inviscid shock profile of $15 \mathrm{~mm}$ height square-faced protuberance with that of $30 \mathrm{~mm}$ diameter cylinder of similar height, both having close values of $R_{N}$. The $R_{N}$ for the square-faced and the $30 \mathrm{~mm}$ diameter cylindrical protuberance was $31.28 \mathrm{~mm}$ and $32.37 \mathrm{~mm}$ respectively. The nose of the shocks are made to coincide in the figure so as to make a comparison between them. Lines on the two shock profiles are marked in order to locate the sonic point on them. The difference in the spanwise extent of the sonic points is minimal, owing to the little difference in $R_{N}$ of the two inviscid shocks. As can be observed from the figure, the profile especially in the strong shock region, matches very well for the two different geometries having similar $R_{N}$.

The separation length was previously shown to be correlated with the strong shock region through the inviscid shock parameters $\Delta_{S}$ and $w_{s}$ (figure 30). However, the correlation is Mach number dependent. Further, it offers very little scope for physical interpretation beyond the significance of strong shock portion in fixing separation location, and does not illustrate 


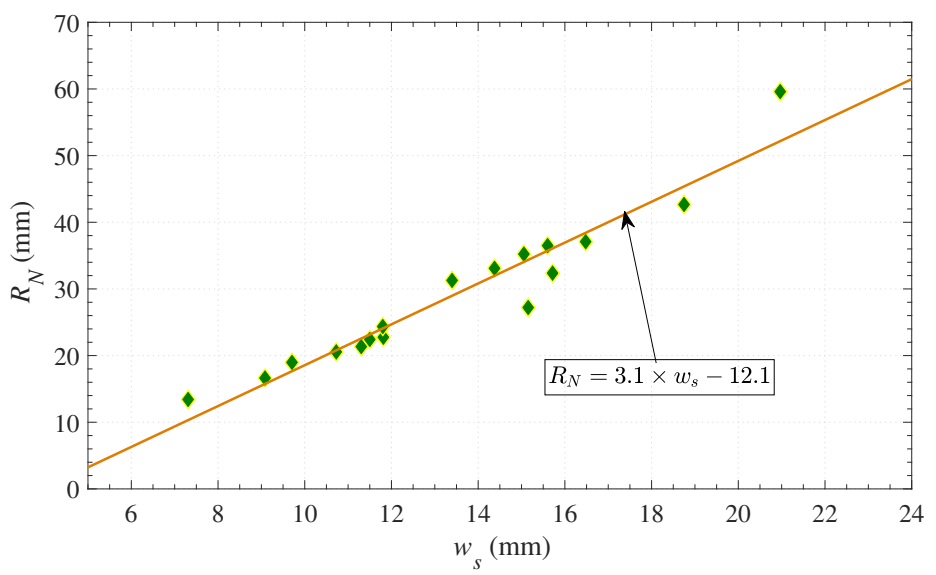

Figure 33: Variation of $R_{N}$ with $w_{s}$ for all the geometries and Mach numbers considered in the study.

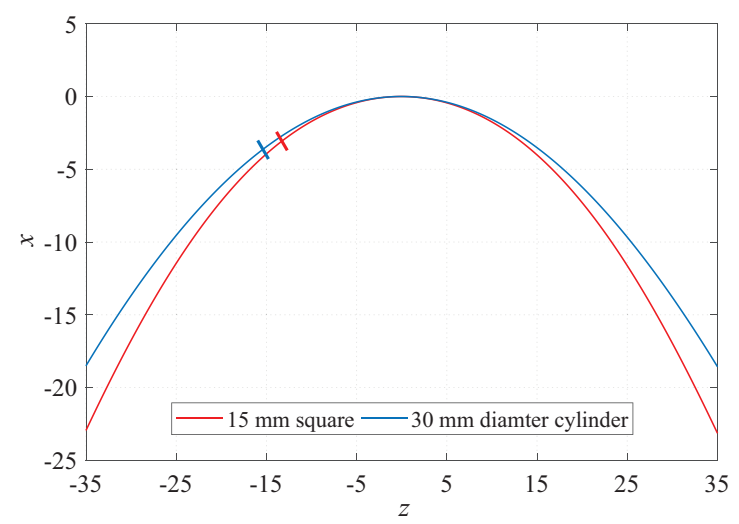

Figure 34: Comparison of inviscid shock for $15 \mathrm{~mm}$ square-faced protuberance and cylindrical (30 $\mathrm{mm}$ diameter and $15 \mathrm{~mm}$ height) protuberance. The lines on the inviscid shock marks the sonic point location on the respective shocks.

the physical phenomena, unlike the 2-dimensional scaling laws/correlations which have a theoretical basis. By considering the similarities in centreline surface pressure distribution between protuberance induced SBLI and 2-dimensional SBLI, and the significance of the 'strong' bow shock portion in fixing $L_{s e p}$, it is reasonable to expect that the $L_{s e p}$ for protuberances can be expressed as a modification to the length of the plateau region in 2-dimensional separation brought about by the relieving effect in the vicinity of the centreline. We thus seek to modify the 2-dimensional scaling with the introduction of $R_{N}$, which in turn is related to the second gradient of pressure jump along the inviscid shock at nose; in other words, obtain a universal scaling which is also applicable to 2-dimensional SBLI when $R_{N}$ tends to infinity. Starting with the correlation presented by Katzer (1989), the pressure term in the correlation is modified with the inclusion of the $R_{N}$, indirectly the pressure variation along the shock. The pressure term in Katzer's correlation is multiplied by $\left(1-\delta_{x} / R_{N}\right)$. The reason for such form is that for 2-dimensional bodies, where $R_{N}$ goes to infinity, $\left(1-\delta_{x} / R_{N}\right)$ equals to one. The plot of the correlation between the non-dimensional separation length (multiplied by cube of Mach number) and the modified 


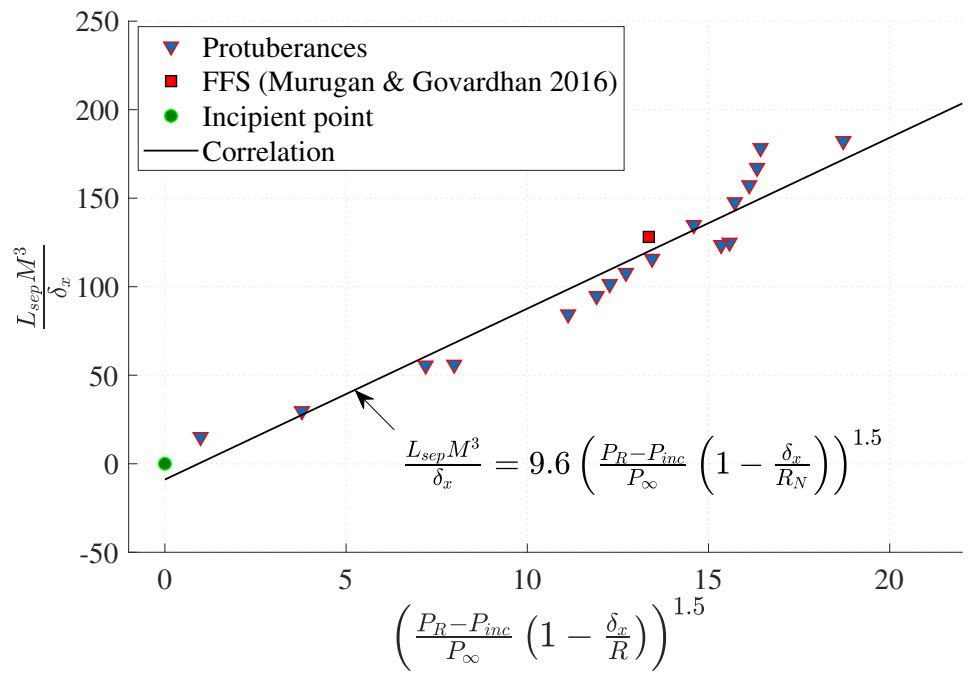

Figure 35: Correlation of non-dimensional separation length (multiplied by cube of Mach number) with the pressure term modified for 3-dimensional protuberances.

pressure term is shown in figure 35. Since $L_{\text {sep }}=0$ for $P_{R}$ is less than or equal to $P_{\text {inc }}$, a point corresponding to $P_{i n c}$ is also added in the figure for reference. A very good correlation with $R^{2}$ value of 0.945 can be seen. The correlation for the 3 -dimensional protuberances is thus given by:

$$
\frac{L_{s e p} M_{\infty}^{3}}{\delta_{x}}=9.6\left(\frac{P_{R}-P_{i n c}}{P_{\infty}}\left(1-\frac{\delta_{x}}{R_{N}}\right)\right)^{1.5}
$$

A small negative intercept value of 8.9 can be observed, but it is less than the RMS of the deviation of the data (value of 12) from the line fit. A non-linear relation of the $L_{s e p}$ with the pressure term is obtained for the 3-dimensional SBLI due to protuberances. Interestingly, the exponent of the pressure term is obtained to be 1.5 , which is the same as that in the 2-dimensional theoretical scaling laws. This is in contrast to the linear pressure term in the correlations for 2-dimensional laminar SBLI based on experimental and computational data, as mentioned previously. In order to show the applicability of the correlation 4.8 for 2-dimensional cases, experimental data for Mach 2.54 turbulent flow over forward-facing step was taken from the study by Murugan \& Govardhan (2016) and substituted in the correlation. As can be seen from the figure 35, the corresponding point falls close to the correlation. Thus, the equation 4.8 serves as a universal correlation for separation length for turbulent SBLI due to 2-dimensional and 3-dimensional protuberances with height comparable to or greater than the boundary layer thickness. It also expresses the essential physics concerning shock induced separation. The 2-dimensional scaling law is obtained theoretically by the integration of 'triple deck' equations; it explains the effect of flow parameters like Reynolds number and shock strength on the separation length. As a modification to the 2-dimensional scaling law, the present correlation introduces and explains the influence of the relieving effect in the vicinity of centreline. In 3-dimensional protuberances, the mid-span flow field is thus interpreted to be similar to the 2-dimensional SBLI, with a scaling factor for separation length introduced by the relieving effects close to mid-span.

Typically, for the correlations concerning SBLI due to compression corners, the $L_{\text {sep }}$ is taken as the distance from the corner to the separation location; in such cases the inviscid shock foot location is the same as the corner position. However, in the present cases, the shock is detached. Therefore the distance between the nose of the inviscid shock and the separation position is $L_{s e p}-\Delta_{s}$. Figure 36 shows the correlation with the modified separation length. The correlation 


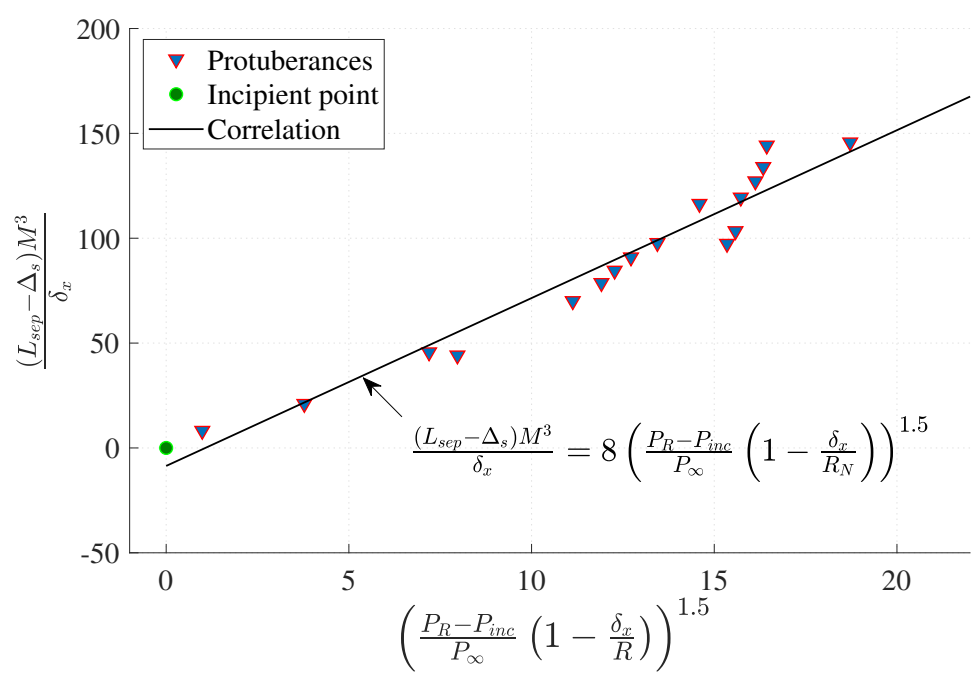

Figure 36: Correlation of the modified non-dimensional separation length with pressure term for various test cases in the study.

obtained is given as:

$$
\frac{\left(L_{\text {sep }}-\Delta_{S}\right) M_{\infty}^{3}}{\delta_{x}}=8\left(\frac{P_{R}-P_{\text {inc }}}{P_{\infty}}\left(1-\frac{\delta_{x}}{R_{N}}\right)\right)^{1.5}
$$

The correlation is not very different from equation 4.8. The $R^{2}$ value of the fit of the data with the correlation is 0.952 . The RMS value of the deviation from the correlation is 9.6; hence, a better agreement of the protuberance data point with the correlation is achieved upon modifying the separation length. Again, a small negative intercept (8.5) can be observed which falls in the error range of the correlation, thus can be ignored. The correlation 4.9 takes into consideration the effects of various geometrical parameters and incoming flow parameters through the $R_{N}$ term. The Reynolds number does not explicitly appear in the proposed correlations. Since the incoming boundary layer is turbulent, the exponent over Reynolds number in the scaling law is expected to be $1 / 5$, as discussed before; the dependence on the term is thus very weak. The literature on protuberance induced SBLI too (Sedney \& Kitchens Jr 1977; Price Jr \& Stallings Jr 1967; Young et al. 1968; Korkegi 1971; Souverein et al. 2013) suggest weak dependence of $L_{\text {sep }}$ on Reynolds number for turbulent flows. The Reynolds number effects, however, indirectly feature in the correlation through the boundary layer thickness $\delta_{x}$.

The position of the horseshoe vortex core was found to increase with the height of the protuberance. Figures 37(a) and 37(b) show the variation of streamwise distance of the vortex core from the front face of the protuberance $\left(x_{v o r}\right)$ and wall-normal distance of the vortex core $\left(y_{v o r}\right)$ with the height, respectively for cylindrical and rectangular-faced protuberances. It can be seen from the figure that the slope of both the curves is progressively diminishing and the distances $\left(x_{v o r}\right.$ and $\left.y_{v o r}\right)$ appears to be approaching an asymptotic value as the height is increased. The reason for this trend can be attributed to the previous observation that $L_{s e p} / D$ itself approaches an asymptotic value when $h / D$ is varied (figure 20). It is interesting to observe that $y_{\text {vor }}$ too increases with the height of the protuberance, along with $x_{v o r}$. With increasing $h$, the extent of the separation bubble increases, which results in more fluid mass getting entrained by the primary vortex. To accommodate this increment in fluid mass to recirculate around the vortex core, $y_{\text {vor }}$ increases. The positions of the horseshoe vortex for cylindrical and rectangular-faced protuberances are also found to be linearly related to $L_{\text {sep }} / h$, as shown in figure 37 (c). Thus, the vortex core position too 


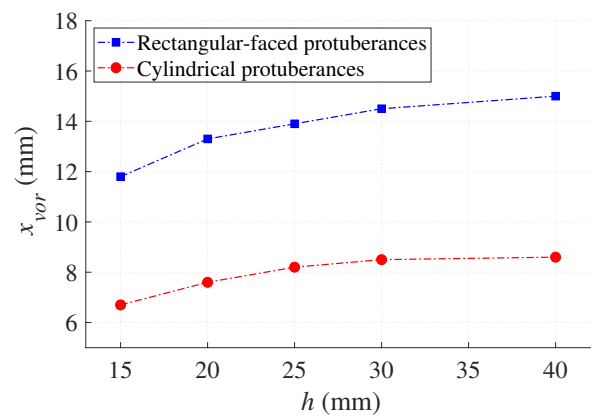

(a)

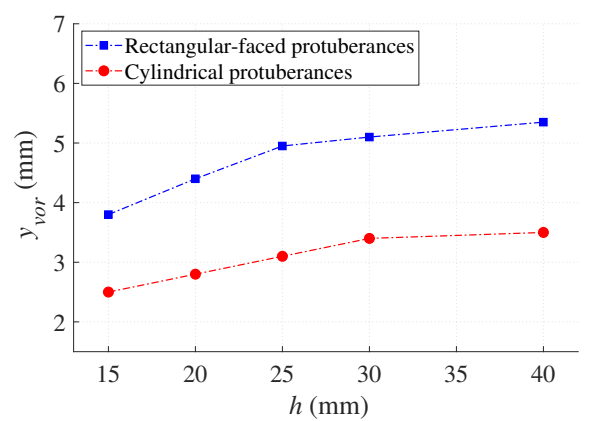

(b)

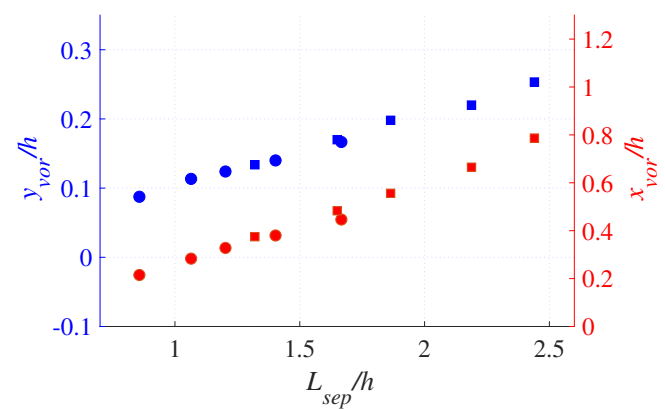

(c)

Figure 37: Dependence of (a) $x_{v o r}$ on $h$ and (b) $y_{v o r}$ on $h$. (c) Plot of $y_{v o r} / h$ and $x_{v o r} / h$ variation with $L_{s e p} / h$. The square marker identifies rectangular-faced protuberances, while the circular marker represents cylindrical protuberances.

depends on the inviscid shock and other flow parameters in the same way the separation length does.

However, the profile of the separation shock seems to be dependent on more parameters. The radius of curvature of the separation shock $\left(R_{\text {sep }}\right)$ was found to vary linearly with the radius of curvature of the inviscid shock $\left(R_{N}\right)$, for a given protuberance shape. Figure 38 shows the linear relation between the radius of curvatures of the two shocks for cylindrical and rectangular-faced protuberances. Both the dimensions are normalized with the height of the protuberance. It can be seen that the line fits for cylindrical and rectangular-faced protuberances are different. While the $L_{\text {sep }}$ is only dependent on the centreline pressure rise and the reliving effect in the vicinity of centreline, the $R_{\text {sep }}$ and thus, the shape of the separation shock could be also influenced by the overall shape of the inviscid bow shock including the weak shock portion.

\section{Conclusions}

Shockwave-turbulent boundary layer interactions due to 3-dimensional protuberances with heights larger than the local boundary layer thickness were investigated with the help of wind tunnel experiments and RANS computations. The computations were validated with the experimental data at freestream Mach number of 2.89 from the present study, as well as with the available data from the literature for other Mach numbers. Using extensive computational data, a detailed parametric study was performed by varying Mach number, protuberance shape and dimensions. When compared with the 2-dimensional shock boundary layer interaction (SBLI), 


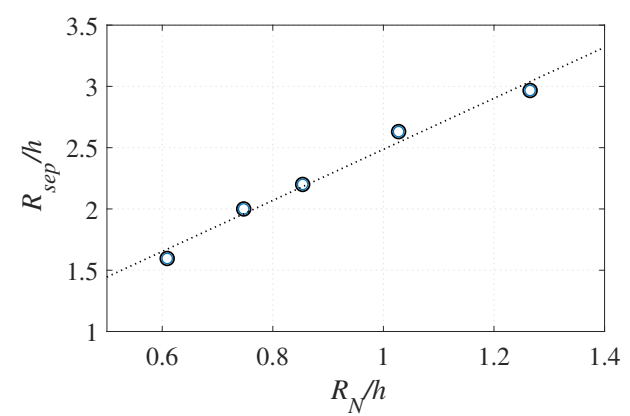

(a)

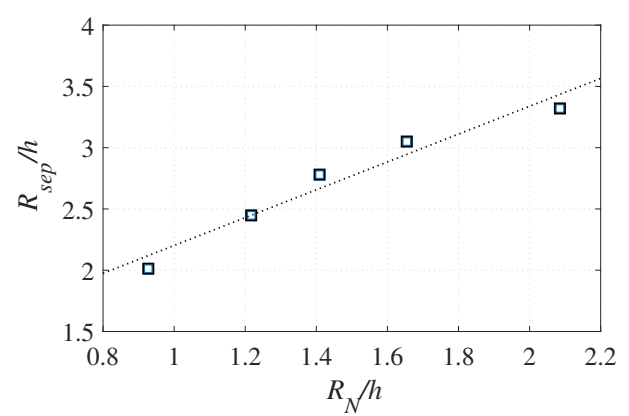

(b)

Figure 38: $R_{\text {sep }}$ variation with $R_{N}$ for (a) cylindrical protuberances and (b) rectangular-faced protuberance. The two parameters are non-dimensionalized with respective heights of the protuberance.

the surface pressure distribution for the protuberance induced SBLI exhibited spanwise variations in pressure, with a distinctive low-pressure region downstream of the plateau pressure region. On the protuberances front surface too, there is a low-pressure region between the reattachment point and the corner at base plate. These are also regions in the recirculating flow near which localized supersonic flow pockets are observed and are related to the relieving effect. A horseshoe vortex is formed by the separated streamlines at the mid-span and its close neighbourhood, which are below the streamlines reattaching on the protuberance. Despite these differences with the 2-dimensional SBLI, the mid-span surface pressure variation for the protuberance induced SBLI exhibited striking similarities with the surface pressure distribution for 2-dimensional SBLI. The plateau pressure was found to be independent of the geometry of the protuberance and could be predicted using the free interaction theory for 2-dimensional SBLI. The separation line and the mid-span separation length $L_{\text {sep }}$ are however dependent on the shape of the protuberance. $L_{\text {sep }}$ was however observed to be strongly correlated with the extent of the strong shock portion of the inviscid bow shock.

The reattaching streamlines encountered a normal shock before the protuberance, nearly stagnating and achieving a peak pressure as it reattaches on the protuberance surface. Since the streamlines immediately below the reattachment turn back towards the wall, move close to the surface and reach the mid-span separation location on the wall, the spanwise extent of the reattachment region on the protuberance plays a significant role in determining the mid-span separation length $L_{\text {sep. }}$. It is in turn related to the relieving effect close to the mid-span, where the bow shock is strong. The relieving effect in the strong shock portion is characterized by a single parameter, which is the nose radius of curvature on the wall plane of the inviscid bow shock, $R_{N}$. Using this parameter, a scaling for $L_{\text {sep }}$ is proposed by modifying the pressure term in the scaling law (Katzer 1989) for 2-dimensional laminar SBLI. Based on the data from the present study, the following correlation relating mid-span separation length with upstream flow parameters and $R_{N}$ (characterizing the relieving effect in strong shock region) was obtained:

$$
\frac{L_{\text {sep }} M_{\infty}^{3}}{\delta_{x}}=9.6\left(\frac{P_{R}-P_{i n c}}{P_{\infty}}\left(1-\frac{\delta_{x}}{R_{N}}\right)\right)^{1.5}
$$

The correlation also closely predicted the separation length for 2-dimensional normal shock induced turbulent separation due to forward facing step (data taken from Murugan \& Govardhan (2016)), for which $R_{N} \rightarrow \infty$. The streamwise distance of the horseshoe vortex core from the 
protuberance as well as its vertical distance from the base plate were found to be linearly related to $L_{\text {sep }}$.

\section{Acknowledgement}

The authors would like to thank Prof. G. Rajesh and his group at the Department of Aerospace Engineering, IIT Madras, for kindly letting us use some of their experimental equipment for this study. In addition, the authors would like to thank Mr. K. Ramachandra, Mr. V. Siva, Mr. G. Sivaprasad and Mr. M. Divakaran from the Department of Aerospace Engineering, IIT Madras, for their assistance in carrying out experiments. The research work is supported by the Science and Engineering Research Board of the Department of Science and Technology, Government of India, SERB grant no. SRG/2019/001793.

\section{REFERENCES}

BelotserkovSKiI, O. M. 1959 Flow past a circular cylinder with a detached shock wave. Tech. Rep.. Research and Advanced Development Div., Avco Corp., Wilmington, MA, RAD-9-TM-59-66.

BILLIG, F. S. 1967 Shock-wave shapes around spherical-and cylindrical-nosed bodies. Journal of Spacecraft and Rockets 4 (6), 822-823.

Burbank, P. B., Newlander, R. A. \& Collins, I. K. 1962 Heat transfer measurements on attached protruberances in a supersonic flow turbulent boundary layer at mach numbers $2.65,3.51$, and 4.44 . Tech. Rep.. NASA TN-D-1372.

BURGGRAF, O. R. 1975 Asymptotic theory of separation and reattachment of a laminar boundary layer on a compression ramp. Tech. Rep.. Ohio State University Research Foundation, AGARD-CP 168.

CAPPELLI, D. \& MANSOUR, N. N. 2013 Performance of reynolds averaged navier-stokes models in predicting separated flows: study of the hump flow model problem. In 31st AIAA Applied Aerodynamics Conference, pp. 31-54.

Chapman, D. R., Kuehn, D. M. \& Larson, H. K. 1958 Investigation of separated flows in supersonic and subsonic streams with emphasis on the effect of transition. Tech. Rep.. NACA-TR-1356.

Clemens, N. T. \& Narayanaswamy, V. 2014 Low-frequency unsteadiness of shock wave/turbulent boundary layer interactions. Annual Review of Fluid Mechanics 46, 469-492.

Davis, J. P. \& Sturtevant, B. 2000 Separation length in high-enthalpy shock/boundary-layer interaction. Physics of Fluids 12 (10), 2661-2687.

DÉLERY, J. \& MARvin, J. G. 1986 Shock-wave boundary layer interactions. Tech. Rep.. AGARD 280.

Dolling, D. S. 2001 Fifty years of shock-wave/boundary-layer interaction research: what next? AIAA Journal 39 (8), 1517-1531.

Dolling, D. S. \& Bogdonoff, S. M. 1981 Scaling of interactions of cylinders with supersonic turbulent boundary layers. AIAA Journal 19 (5), 655-657.

Dussauge, J. P. \& PiPONNiAu, S. 2008 Shock/boundary-layer interactions: Possible sources of unsteadiness. Journal of Fluids and Structures 24 (8), 1166-1175.

DéLERY, JEAN 2011 Physical introduction. In Shock Wave-Boundary-Layer Interactions (ed. H. Babinsky \& J. K. Harvey), pp. 5-86. Cambridge University Press.

EDNEY, B. E. 1968 Effects of shock impingement on the heat transfer around blunt bodies. AIAA Journal 6 (1), 15-21.

HAHN, P. V. \& FRENDI, A. 2013 Interaction of three-dimensional protuberances with a supersonic turbulent boundary layer. AIAA Journal 51 (7), 1657-1666.

Humble, R. A., Elsinga, G. E., Scarano, F. \& Van Oudheusden, B. W. 2009 Three-dimensional instantaneous structure of a shock wave/turbulent boundary layer interaction. Journal of Fluid Mechanics 622, 33.

Hung, C. M. \& Buning, P. G. 1985 Simulation of blunt-fin-induced shock-wave and turbulent boundarylayer interaction. Journal of Fluid Mechanics 154, 163-185.

KATZER, E. 1989 On the lengthscales of laminar shock/boundary-layer interaction. Journal of Fluid Mechanics 206, 477-496.

Knight, D., Yan, H., Panaras, A. G. \& Zheltovodov, A. 2003 Advances in cfd prediction of shock wave turbulent boundary layer interactions. Progress in Aerospace Sciences 39 (2-3), 121-184. 
KoLÁř, V. 2007 Vortex identification: New requirements and limitations. International journal of heat and fluid flow 28 (4), 638-652.

KORKEGI, R. H. 1971 Survey of viscous interactions associated with high mach number flight. AIAA Journal 9 (5), 771-784.

LAKSHMANAN, B. \& TIWARI, S. N. 1994 Investigation of three-dimensional separation at wing/body junctions in supersonic flows. Journal of aircraft 31 (1), 64-71.

Lindörfer, S. A., Combs, C. S., Kreth, P. A., Bond, R. B. \& Schmisseur, J. D. 2020 Scaling of cylindergenerated shock-wave/turbulent boundary-layer interactions. Shock Waves pp. 1-13.

Lindörfer, S. A., Combs, C. S., Kreth, P. A. \& Schmisseur, J. D. 2017 Numerical simulations of a cylinder-induced shock wave/boundary layer interaction. In 55th AIAA Aerospace Sciences Meeting, p. 0534.

Moffat, R. J. 1988 Describing the uncertainties in experimental results. Experimental thermal and fluid science 1 (1), 3-17.

Murugan, J. N. \& GovardHAN, R. N. 2016 Shock wave-boundary layer interaction in supersonic flow over a forward-facing step. Journal of Fluid Mechanics 807, 258-302.

Ozawa, H. \& LaURence, S. J. 2018 Experimental investigation of the shock-induced flow over a wallmounted cylinder. Journal of Fluid Mechanics 849, 1009-1042.

Ozcan, O. \& Holt, M. 1984 Supersonic separated flow past a cylindrical obstacle on a flat plate. AIAA Journal 22 (5), 611-617.

Price Jr, E. A. \& Stallings JR, R. L. 1967 Investigation of turbulent separated flows in the vicinity of fin-type protuberances at supersonic mach numbers. Tech. Rep.. NASA TN-D-3804.

Rizzetta, D. P., Burggraf, O. R. \& Jenson, R. 1978 Triple-deck solutions for viscous supersonic and hypersonic flow past corners. Journal of Fluid Mechanics 89 (3), 535-552.

SEDNEY, R. 1973 A survey of the effects of small protuberances on boundary-layer flows. AIAA Journal $11(6), 782-792$.

Sedney, R. \& Kitchens JR, C. W. 1975 The structure of three-dimensional separated flows in obstacleboundary layer interactions. Tech. Rep.. Army Ballistic Research Lab, AGARD-CP-168.

SEDNEy, R. \& Kitchens JR, C. W. 1977 Separation ahead of protuberances in supersonic turbulent boundary layers. AIAA Journal 15 (4), 546-552.

Souverein, L. J., BAKKER, P. G. \& DUPONT, P. 2013 A scaling analysis for turbulent shock-wave/boundarylayer interactions. Journal of Fluid Mechanics 714, 505-535.

SRIRAM, R. \& JAGADEESH, G. 2015 Correlation for length of impinging shock-induced large separation bubble at hypersonic speed. AIAA Journal 53 (9), 2771-2776.

Sriram, R., Srinath, L., DevaraJ, M. K. K. \& Jagadeesh, G. 2016 On the length scales of hypersonic shock-induced large separation bubbles near leading edges. Journal of Fluid Mechanics 806, 304-355.

Stewartson, K. \& Williams, P. G. 1969 Self-induced separation. Proc. of the Royal Society of London. A. 312 (1509), 181-206.

SURBER, T. E. 1965 Heat transfer in the vicinity of surface protuberances. Journal of Spacecraft and Rockets 2 (6), 978-980.

TruitT, R. W. 1965 Hypersonic turbulent boundary-layer interference heat transfer in vicinity of protuberances. AIAA Journal 3 (9), 1754-1755.

Voitenko, D. M., Zubkov, A. I. \& Panov, Y. A. 1966 Supersonic gas flow past a cylindrical obstacle on a plate. Fluid Dynamics 1 (1), 84-88.

Voitenko, D. M., Zubkov, A. I. \& Panov, Y. A. 1969 Supersonic flow past an inclined obstacle. Fluid Dynamics 4 (6), 99-103.

WANG, D., ZhaO, Y., XIA, Z., WANG, Q. \& HuANG, L. 2012 Experimental investigation of supersonic flow over a hemisphere. Chinese Science Bulletin 57 (15), 1765-1771.

WeSTKAEMPER, J.C. 1968 Turbulent boundary-layer separation ahead of cylinders. AIAA Journal 6 (7), $1352-1355$.

Wu, M. \& Martin, M. P. 2008 Analysis of shock motion in shockwave and turbulent boundary layer interaction using direct numerical simulation data. Journal of Fluid Mechanics 594, 71.

YAO, J. \& Hussain, F. 2018 Toward vortex identification based on local pressure-minimum criterion in compressible and variable density flows. Journal of Fluid Mechanics 850, 5-17.

Young, F. L., KAUfMAN, L. G., KorKegi, R. H. \& OTHERS 1968 Experimental investigation of interactions between blunt fin shock waves and adjacent boundary layers at mach numbers 3 and 5. Tech. Rep.. ARL 68- 0214. 